\title{
Atomic data for the X-ray lines of Fe VIII and Fe IX ${ }^{\star}$
}

\author{
B. O’Dwyer ${ }^{1}$, G. Del Zanna ${ }^{1}$, N. R. Badnell ${ }^{2}$, H. E. Mason ${ }^{1}$, and P. J. Storey ${ }^{3}$ \\ ${ }^{1}$ DAMTP, Centre for Mathematical Sciences, Wilberforce Road, Cambridge CB3 0WA, UK \\ e-mail: b.o-dwyer@damtp.cam.ac.uk \\ 2 Department of Physics, University of Strathclyde, Glasgow G4 0NG, UK \\ 3 Department of Physics and Astronomy, University College London, Gower Street, London WC1E 6BT, UK
}

Received 22 August 2011 / Accepted 19 September 2011

\section{ABSTRACT}

\begin{abstract}
The distorted wave extension of the AUTOSTRUCTURE code has been used to calculate energy levels, radiative transition probabilities and collisional excitation rates of Fe vIII and Fe IX up to $n=6$ for Fe IX and $n=7$ for Fe vIII. We have compared some of the data with previous calculations, finding overall agreement for radiative transition rates, but interesting differences for some collisional data. We have merged our data for the higher energy levels with published R-matrix collisional excitation rates for the lower ones to calculate spectral line intensities and compare them with observations. In particular, we have focused on the transitions from high energy levels of Fe VIII \& Fe IX which are present in the 93-95 Å region. A few new identifications are tentatively provided. We find that Fe Ix 5f-3d and Fe VIII 7f-3d transitions only comprise a small fraction of the observed lines in the 93-95 A region for quiet Sun conditions, and thus their contribution to the Solar Dynamics Observatory (SDO) Atmospheric Imaging Assembly (AIA) 94 Å band is expected to be small.
\end{abstract}

Key words. atomic data - line: identification - techniques: spectroscopic

\section{Introduction}

The 50-170 A spectral region is dominated by a forest of transitions, many of which are from $n=4,5,6$ and 7 states of highly ionised iron ions, and for which little atomic data are available (see Fawcett et al. 1968; Jordan 1968). This spectral region has been observed with various solar instruments on-board sounding rockets, and more recently with Chandra LETG (see Brinkman et al. 2000) and the Solar Dynamics Observatory (SDO). SDO has been providing new extreme-ultraviolet (EUV) observations of the Sun, at unprecedented cadence. One of the instruments, the Atmospheric Imaging Assembly (AIA, see Lemen et al. 2011) has been providing narrow-band EUV images at various wavelengths, centred at 94,131, 171, 193, 211, and $335 \AA$. The relevant atomic data for the spectral lines contributing to some of these bands are relatively well understood, however a significant amount of data is still lacking. See O'Dwyer et al. (2010) and Del Zanna et al. (2011) for details.

This work is in part motivated by the desire to determine the contribution of transitions from high energy levels of Fe viII \& Fe Ix to the SDO AIA $94 \AA$ band. The work of Aschwanden \& Boerner (2011) has hinted at the presence of missing flux at lower temperatures in the response curve for the $94 \AA$ band. Fe vIII 7f-3d transitions at 93.469 and $93.616 \AA$ have been identified by Ramonas \& Ryabtsev (1980). Approximate excitation data for these transitions were estimated by Czyzak \& Krueger (1966), and a proper scattering calculation was needed. The presence of Fe Ix $5 \mathrm{f}-3 \mathrm{~d}$ transitions in the $94 \AA$ region has been

* A complete list of calculated energies, radiative data and thermally averaged collision strengths is only available at the CDS via anonymous ftp to cdsarc.u-strasbg. fr $(130.79 .128 .5)$ or via http://cdsarc.u-strasbg.fr/viz-bin/qcat?J/A+A/537/A22 proposed by Lepson et al. (2002), but appropriate calculations have been missing.

In this work, we have therefore carried out calculations for Fe vIII and Fe IX using a new distorted wave (DW) development of the AUTOSTRUCTURE code, described in Badnell (2011) and outlined below. We have used this new code to obtain collisional and radiative data for levels up to $n=6$ for Fe IX and $n=7$ for Fe viII. We have merged the data for the higher energy levels with those of the lower R-matrix ones $(n=3)$, previously available, to build an atomic model and calculate spectral line intensities, to compare with observations. Recently, Foster \& Testa (2011) have also carried out atomic calculations for the Fe Ix 5f-3d transitions using the Flexible Atomic Code (FAC, see $\mathrm{Gu}$ 2003). Towards the end of the paper, we briefly compare our results with theirs.

The discussion in this paper focuses on transitions from $n=4-7$ configurations observed in the Soft X-rays, from 50 to $170 \AA$ A. These atomic data are needed, because there are a large number of lines (many of which are still unidentified) in this wavelength range. This spectral range is also routinely observed now by the SDO Extreme ultraviolet Variability Experiment (EVE). EVE includes two spectrographs and multiple photometers for measuring the solar EUV irradiance from 1 to $1220 \AA$ (Woods et al. 2010). The EUV spectra are from two Multiple EUV Grating Spectrographs (MEGS) in the 50-380 ̊ and 3501050 Å spectral ranges, with $\simeq 1$ A spectral resolution.

The paper is organised as follows. In Sect. 2, we give details of the methods used for the atomic structure and electron scattering calculations. The Fe Ix and $\mathrm{Fe}$ VIII calculations are discussed in Sects. 3 and 4, respectively. Comparisons are made with the results of previous publications. In Sect. 5 we benchmark the atomic data we have calculated for Fe VIII and Fe IX against solar and laboratory spectra. Our conclusions are given in Sect. 6. 


\section{Method}

The atomic structure calculations were carried out using the program AUTOSTRUCTURE (Badnell 1997) which constructs target wavefunctions using radial wavefunctions calculated in a scaled Thomas-Fermi-Dirac statistical model potential. The scaling parameters were determined by minimising the sum of the energies of all the target terms, computed in LS-coupling, i.e. neglecting all relativistic effects.

The Breit-Pauli distorted wave calculations were carried-out using the autostructure code. Full details of this recent development of the code are found in Badnell (2011). Although it bears some superficial similarities with the historic UCL-DW code (Eissner 1998) due to their common SUPERSTRUCTURE heritage (Eissner et al. 1974), it is an independent implementation which differs fundamentally from the UCL-DW code in a number of important ways. We note salient points of the implementation. It places no restrictions of the atomic structure. Two-body fine-structure can be included if desired, for example. The continuum distorted waves are calculated using the same form for the distorting potential as specified for the target, but now for the $(N+1)$-electron problem. Non-relativistic or kappaaveraged relativistic orbitals can be used. Unlike the boundfree problem (e.g. autoionization) the continuum orbitals are not orthogonalized to the bound orbitals, nor are the "zero" overlaps neglected. Rather, the appropriate exchange overlap integrals are determined. The electrostatic and, optionally, two-body non-fine-structure $(N+1)$-electron interaction Hamiltonian for the collision problem is determined in an unmixed LS-coupling representation. It is then transformed to an LSJ representation. The two-body fine-structure collisional interaction can be added, optionally, at this stage. The full $(N+1)$-electron interaction Hamiltonian is transformed to a full Breit-Pauli $\mathrm{jK}$-coupling representation (i.e. including both configuration and fine-structure target mixing) in the same manner as is done for the (innerregion) Breit-Pauli R-matrix.

Collision strengths are calculated at the same set of final scattered energies for all transitions. Zero gives all threshold transitions, for example. A small continuum interpolation basis is used to enable the interaction Hamiltonian to be evaluated at the appropriate initial scattering energy for each transition. "Top-up" in the contribution of high partial waves is done using the same Breit-Pauli methods and subroutines implemented in the R-matrix outer-region code STGF. The implementation is designed for efficiency in determining collision strengths from a low-lying set of (user specifiable) "metastable" levels to all possible "spectroscopic" excited states. "Correlation" levels/terms/configurations can be flagged for efficiency so that they contribute only to the target description. The final deliverable is a (type-5) adf04 file (Summers 2006) which contains energy levels, radiative rates, ordinary collision strengths (as a function of final scattered energy) and infinite energy Born limits (and line strengths). These ordinary collision strengths can then be convoluted with any desired electron energy distribution. A utility code (adf04_om2ups.f) is provided which converts this file to the more familiar (type-3) adf04 file which contains Maxwellaveraged effective collision strengths.

We note that resonances are omitted in the first instance. Since resonances in electron-impact excitation are just the complement of dielectronic recombination then their contribution can be determined independently, if desired. Alternatively, our approach lends itself to complementing the results of an Rmatrix calculation, as herein, since resonances are more prominent in low-lying transitions. There is also compatibility in atomic structure which aids merging of the two datasets. Starting with the R-matrix target structure, which may be much larger than that for which collision data is calculated, additional orbitals and configurations included in the DW calculation should not perturb the original structure too much. If it does then the original R-matrix structure is questionable.

In addition to the original tests and comparisons (Badnell 2011) some further comparisons of collision strengths from the AUTOSTRUCTURE DW code have been made with both FAC and (background) R-matrix collision strengths, and which emphasise the importance of the atomic structure in such - see Liang \& Badnell (2011) and Liang et al. (2011).

\section{FeIX}

A significant number of transitions from $n=4,5$ levels for this ion remain unidentified. Kruger et al. (1937) identified the two strong decays from the $4 \mathrm{~s}$ levels to the ground state. Alexander et al. (1965) later identified four decays from the $4 \mathrm{~d}$ and $5 \mathrm{~s}$ levels. Wagner \& House (1971) were the first to identify 12 transitions from the $3 \mathrm{p}^{5} 3 \mathrm{~d}-3 \mathrm{p}^{5}$ 4f array, in the 111.69-116.81 spectral range. We adopt the measurements from Fawcett et al. (1972) who revised the Wagner \& House (1971) wavelengths. Young (2009) identified three lines from the $3 \mathrm{~s}^{2} 3 \mathrm{p}^{4} 3 \mathrm{~d}^{2}$ configuration and one from the $3 s^{2} 3 p^{5} 4 p$ configuration. Young \& Landi (2009) identified some more transitions from the $3 \mathrm{~s}^{2} 3 \mathrm{p}^{4}$ $3 \mathrm{~d}^{2}$ configuration. Landi \& Young (2009) identified two more transitions from the $3 \mathrm{~s}^{2} 3 \mathrm{p}^{5} 4 \mathrm{p}$ configuration.

Lines from Fe Ix have been observed in the extreme ultraviolet with an electron beam ion trap (EBIT) by Beiersdorfer et al. (1999) and Lepson et al. (2002). Lepson et al. (2002) suggested that a few weak lines observed around $94 \AA$ arise from $5 f-3 d$ transitions, however a firm identification was not provided.

Radiative data for this ion have been calculated by a number of authors. Aggarwal et al. (2006) calculated radiative data with the General purpose Relativistic Atomic Structure Package (GRASP) for transitions between some $n=3$ and the $3 \mathrm{~s}^{2} 3 \mathrm{p}^{5} 4 l$ configurations.

Verma et al. (2006) produced a large-scale structure calculation using Hibbert's CIV3 Program and semi-empirical corrections to obtain a good match in level energies for those known. They included some $n=3,4,5$ configurations, but not the $3 \mathrm{~s}^{2}$ $3 \mathrm{p}^{5}$ 5f. The configuration $3 \mathrm{~s}^{2} 3 \mathrm{p}^{3} 3 \mathrm{~d}^{3}$, which is important for configuration interaction, was omitted from their calculations.

FAC atomic structure calculations were carried out by Landi $\&$ Young (2009) up to $n=4$.

In terms of electron excitation data, the most accurate calculation so far is from Storey et al. (2002). These data are included in CHIANTI v.6 (Dere et al. 2009). Storey et al. (2002) performed an R-matrix calculation, producing collisional excitations rates among the lowest 140 energy levels, in six configurations, which include levels from the $3 \mathrm{~s}^{2} 3 \mathrm{p}^{5} 4 \mathrm{~s}$ and $3 s^{2} 3 p^{5} 4 p$ configurations. His target had 12 configurations, of which 6 were included as correlation configurations.

We have run several structure calculations with various configurations (12, 27, 45 and 67 configuration calculations). The 12 configuration target is identical to that of Storey et al. (2002). Our final choice for the scattering calculation is the largest run, where the target wavefunctions are expanded in the 67 configuration basis listed in Table 1 . Those configurations listed above the horizontal line in Table 1 are spectroscopic configurations. Those below this line are present solely for correlation purposes. The Thomas-Fermi-Dirac scaling parameters are also given in Table 1. 
Table 1. Electron configuration basis for the DW calculation and orbital scaling parameters for Fe IX.

\begin{tabular}{|c|c|c|c|c|c|}
\hline \multicolumn{2}{|c|}{ Configurations } & \multicolumn{4}{|c|}{ Scaling parameters } \\
\hline even & odd & & & & \\
\hline $3 s^{2} 3 p^{6}$ & $3 s^{2} 3 p^{5} 3 d$ & 1s: & 1.4171 & $2 \mathrm{~s}:$ & 1.1241 \\
\hline $3 \mathrm{~s} 3 \mathrm{p}^{6} 3 \mathrm{~d}$ & $3 s 3 p^{5} 3 d^{2}$ & $2 \mathrm{p}:$ & 1.0672 & 3s: & 1.1499 \\
\hline $3 s^{2} 3 p^{4} 3 d^{2}$ & $3 s^{2} 3 p^{5} 4 s$ & $3 \mathrm{p}:$ & 1.1294 & 3d: & 1.1371 \\
\hline $3 s^{2} 3 p^{5} 4 p$ & $3 s^{2} 3 p^{5} 4 d$ & 4s: & 1.1524 & $4 \mathrm{p}:$ & 1.1254 \\
\hline $3 s 3 p^{6} 4 s$ & $3 s^{2} 3 p^{6} 4 p$ & 4d: & 1.1308 & 4f: & 1.2586 \\
\hline $3 \mathrm{~s} 3 \mathrm{p}^{6} 4 \mathrm{~d}$ & $3 \mathrm{~s} 3 \mathrm{p}^{6} 4 \mathrm{f}$ & $5 \mathrm{~s}:$ & 1.1667 & $5 \mathrm{p}:$ & 1.1366 \\
\hline $3 s^{2} 3 p^{5} 4 f$ & $3 s^{2} 3 p^{5} 5 s$ & $5 \mathrm{~d}:$ & 1.1650 & 5f: & 1.7195 \\
\hline $3 \mathrm{p}^{6} 3 \mathrm{~d}^{2}$ & $3 s^{2} 3 p^{5} 5 d$ & 5g: & 1.6330 & 6s: & 1.1935 \\
\hline $3 s^{2} 3 p^{5} 5 p$ & $3 s^{2} 3 p^{5} 5 g$ & $6 \mathrm{p}:$ & 1.1871 & $6 \mathrm{~d}:$ & 1.2061 \\
\hline $3 s^{2} 3 p^{5} 5 f$ & $3 s^{2} 3 p^{5} 6 s$ & 6f: & 1.7170 & 6g: & 1.6577 \\
\hline $3 s^{2} 3 p^{5} 6 p$ & $3 s^{2} 3 p^{5} 6 d$ & & & & \\
\hline $3 s^{2} 3 p^{5} 6 f$ & $3 s^{2} 3 p^{5} 6 g$ & & & & \\
\hline------- & - - - - - - - - - & & & & \\
\hline $3 s^{2} 3 p^{4} 3 d 4 s$ & $3 s^{2} 3 p^{4} 3 d 4 p$ & & & & \\
\hline $3 s^{2} 3 p^{4} 3 d 4 d$ & $3 s^{2} 3 p^{4} 3 d 4 f$ & & & & \\
\hline $3 s 3 p^{4} 3 d^{3}$ & $3 s^{2} 3 p^{3} 3 d^{3}$ & & & & \\
\hline $3 s 3 p^{6} 5 s$ & $3 p^{5} 3 d^{3}$ & & & & \\
\hline $3 \mathrm{~s} 3 \mathrm{p}^{6} 5 \mathrm{~d}$ & $3 s 3 p^{6} 5 p$ & & & & \\
\hline $3 s 3 p^{6} 5 g$ & $3 s 3 p^{6} 5 f$ & & & & \\
\hline $3 s^{2} 3 p^{4} 3 d 5 s$ & $3 s^{2} 3 p^{4} 3 d 5 p$ & & & & \\
\hline $3 s^{2} 3 p^{4} 3 d 5 d$ & $3 s^{2} 3 p^{4} 3 d 5 f$ & & & & \\
\hline $3 s^{2} 3 p^{4} 3 d 5 g$ & $3 \mathrm{~s} 3 \mathrm{p}^{5} 3 \mathrm{~d} 5 \mathrm{~s}$ & & & & \\
\hline $3 s 3 p^{5} 3 d 5 p$ & $3 \mathrm{~s} 3 \mathrm{p}^{5} 3 \mathrm{~d} 5 \mathrm{~d}$ & & & & \\
\hline $3 s 3 p^{5} 3 d 5 f$ & $3 \mathrm{~s} 3 \mathrm{p}^{5} 3 \mathrm{~d} 5 \mathrm{~g}$ & & & & \\
\hline $3 s 3 p^{6} 6 s$ & $3 s 3 p^{6} 6 p$ & & & & \\
\hline $3 s 3 p^{6} 6 d$ & $3 \mathrm{~s} 3 \mathrm{p}^{6}$ 6f & & & & \\
\hline $3 s 3 p^{6} 6 g$ & $3 s^{2} 3 p^{4} 3 d 6 p$ & & & & \\
\hline $3 s^{2} 3 p^{4} 3 d 6 s$ & $3 s^{2} 3 p^{4} 3 d 6 f$ & & & & \\
\hline $3 s^{2} 3 p^{4} 3 d 6 d$ & $3 s 3 p^{5} 3 d 6 s$ & & & & \\
\hline $3 s^{2} 3 p^{4} 3 d 6 g$ & $3 \mathrm{~s} 3 \mathrm{p}^{5} 3 \mathrm{~d} 6 \mathrm{~d}$ & & & & \\
\hline $3 s 3 p^{5} 3 d 6 p$ & $3 \mathrm{~s} 3 \mathrm{p}^{5} 3 \mathrm{~d} 6 \mathrm{~g}$ & & & & \\
\hline $3 \mathrm{~s} 3 \mathrm{p}^{5} 3 \mathrm{~d} 6 \mathrm{f}$ & $3 s 3 p^{5} 3 d 4 s$ & & & & \\
\hline $3 s^{2} 3 p^{2} 3 d^{4}$ & $3 \mathrm{~s} 3 \mathrm{p}^{5} 3 \mathrm{~d} 4 \mathrm{~d}$ & & & & \\
\hline $3 s^{2} 3 p^{4} 4 p^{2}$ & & & & & \\
\hline $3 s 3 p^{5} 3 d 4 p$ & & & & & \\
\hline $3 \mathrm{~s} 3 p^{5} 3 d 4 f$ & & & & & \\
\hline
\end{tabular}

Notes. The configurations below the dashed line are correlation configurations.

In Table 2 we list the calculated and, where available, experimental energies for a sample of levels of the target. A complete list of the calculated energies of all 379 levels of the target is available at the CDS.

Table 3 contains a comparison of transition probabilities for a sample of the stronger transitions. Transition probabilities are provided for our 27, 45 and 67 configuration targets as well as those calculated by Storey et al. (2002) (in agreement with our 12 configuration calculation), Landi (2011, priv. comm.) corresponding to those of Landi \& Young (2009) and Verma et al. (2006). Overall agreement (to within a relative 20\%) is found, with the exception of the $3 \mathrm{p}^{6}{ }^{1} \mathrm{~S}_{0}-3 \mathrm{p}^{5} 5 \mathrm{~s}{ }^{1} \mathrm{P}_{1}$ and $3 \mathrm{p}^{6}{ }^{1} \mathrm{~S}_{0}-3 \mathrm{p}^{5}$ $5 \mathrm{~s}{ }^{3} \mathrm{P}_{1}$ transitions for which there are substantial differences. As Verma et al. pointed out, the $3 \mathrm{~s}^{2} 3 \mathrm{p}^{5} 5 \mathrm{~s}{ }^{1} \mathrm{P}_{1}$ and ${ }^{3} \mathrm{P}_{1}$ are highly mixed, and these levels also mix with the $3 \mathrm{~s} 3 \mathrm{p}^{6} 4 \mathrm{p}^{1} \mathrm{P}_{1}$ level. In our largest structure run, 67 configurations, level 263 $\left(5 \mathrm{~s}{ }^{1} \mathrm{P}_{1}\right)$ is composed of $38 \%\left(5 \mathrm{~s}{ }^{1} \mathrm{P}_{1}\right), 32 \%\left(5 \mathrm{~s}{ }^{3} \mathrm{P}_{1}\right)$ and $10 \%$ (3s $\left.3 \mathrm{p}^{6} 4 \mathrm{p}{ }^{1} \mathrm{P}_{1}\right)$, while level $265\left(5 \mathrm{~s}^{3} \mathrm{P}_{1}\right)$ is composed of $55 \%$ $\left(5 \mathrm{~s}^{3} \mathrm{P}_{1}\right)$, and $41 \%\left(5 \mathrm{~s}^{1} \mathrm{P}_{1}\right)$. Our predicted energy difference between the two 5 s levels $\left(13690 \mathrm{~cm}^{-1}\right)$ is close to the observed one
Table 2. Level energies for Fe IX.

\begin{tabular}{|c|c|c|c|c|}
\hline$i$ & Conf. & Lev. & $E_{\exp }$ & $E_{\text {calc }}$ \\
\hline 1 & $3 s^{2} 3 p^{6}$ & ${ }^{1} \mathrm{~S}_{0}$ & 0.0 & 0.0 \\
\hline 2 & $3 s^{2} 3 p^{5} 3 d$ & ${ }^{3} \mathrm{P}_{0}$ & 405772.0 & $412902(-7130)$ \\
\hline 3 & $3 s^{2} 3 p^{5} 3 d$ & ${ }^{3} \mathrm{P}_{1}$ & 408315.1 & $415718(-7403)$ \\
\hline 4 & $3 s^{2} 3 p^{5} 3 d$ & ${ }^{3} \mathrm{P}_{2}$ & 413669.2 & $421482(-7813)$ \\
\hline 5 & $3 s^{2} 3 p^{5} 3 d$ & ${ }^{3} \mathrm{~F}_{4}$ & 425809.8 & $435212(-9402)$ \\
\hline 6 & $3 s^{2} 3 p^{5} 3 d$ & ${ }^{3} \mathrm{~F}_{3}$ & 429310.9 & $438647(-9336)$ \\
\hline 7 & $3 s^{2} 3 p^{5} 3 d$ & ${ }^{3} \mathrm{~F}_{2}$ & 433818.8 & $443252(-9433)$ \\
\hline 8 & $3 s^{2} 3 p^{5} 3 d$ & ${ }^{3} \mathrm{D}_{3}$ & 455612.2 & $465650(-10038)$ \\
\hline 9 & $3 s^{2} 3 p^{5} 3 d$ & ${ }^{1} \mathrm{D}_{2}$ & 456752.7 & $467102(-10349)$ \\
\hline 10 & $3 s^{2} 3 p^{5} 3 d$ & ${ }^{3} \mathrm{D}_{1}$ & 460616.0 & $470561(-9945)$ \\
\hline 11 & $3 s^{2} 3 p^{5} 3 d$ & ${ }^{3} \mathrm{D}_{2}$ & 462616.6 & $473000(-10383)$ \\
\hline 12 & $3 s^{2} 3 p^{5} 3 d$ & ${ }^{1} F_{3}$ & 465828.4 & $476321(-10493)$ \\
\hline 13 & $3 s^{2} 3 p^{5} 3 d$ & ${ }^{1} \mathrm{P}_{1}$ & 584546 & $601508(-16962)$ \\
\hline 14 & $3 \mathrm{~s} 3 \mathrm{p}^{6} 3 \mathrm{~d}$ & ${ }^{3} \mathrm{D}_{1}$ & 726734 & $737860(-11126)$ \\
\hline 15 & $3 \mathrm{~s} 3 \mathrm{p}^{6} 3 \mathrm{~d}$ & ${ }^{3} \mathrm{D}_{2}$ & 727560 & $738828(-11268)$ \\
\hline 16 & $3 \mathrm{~s} 3 \mathrm{p}^{6} 3 \mathrm{~d}$ & ${ }^{3} \mathrm{D}_{3}$ & 728935 & $740423(-11488)$ \\
\hline 17 & $3 s 3 p^{6} 3 d$ & ${ }^{1} \mathrm{D}_{2}$ & 749871 & $761945(-12074)$ \\
\hline 94 & $3 s^{2} 3 p^{4} 3 d^{2}$ & ${ }^{3} \mathrm{G}_{4}$ & 955790 & $982417(-26627)$ \\
\hline 95 & $3 s^{2} 3 p^{4} 3 d^{2}$ & ${ }^{3} \mathrm{G}_{5}$ & 956322 & $982823(-26501)$ \\
\hline 96 & $3 s^{2} 3 p^{4} 3 d^{2}$ & ${ }^{3} \mathrm{G}_{3}$ & 956787 & $983911(-27124)$ \\
\hline 97 & $3 s^{2} 3 p^{5} 4 s$ & ${ }^{3} \mathrm{P}_{1}$ & 950498 & $989102(-38604)$ \\
\hline 107 & $3 s^{2} 3 p^{5} 4 s$ & ${ }^{1} \mathrm{P}_{1}$ & 965568 & $1004712(-39144)$ \\
\hline 140 & $3 s^{2} 3 p^{5} 4 p$ & ${ }^{1} \mathrm{~S}_{0}$ & 1089949 & $1126230(-36281)$ \\
\hline 196 & $3 s^{2} 3 p^{5} 4 d$ & ${ }^{1} \mathrm{P}_{1}$ & 1198222 & $1240063(-41841)$ \\
\hline 210 & $3 s^{2} 3 p^{5} 4 d$ & ${ }^{3} \mathrm{D}_{1}$ & 1213150 & $1254981(-41831)$ \\
\hline 237 & $3 s^{2} 3 p^{5} 4 f$ & ${ }^{3} \mathrm{D}_{1}$ & 1300923 & $1344314(-43391)$ \\
\hline 238 & $3 s^{2} 3 p^{5} 4 f$ & ${ }^{3} \mathrm{D}_{2}$ & 1302841 & $1346283(-43442)$ \\
\hline 239 & $3 s^{2} 3 p^{5} 4 f$ & ${ }^{3} \mathrm{G}_{5}$ & 1304598 & $1348616(-44018)$ \\
\hline 241 & $3 s^{2} 3 p^{5} 4 f$ & ${ }^{3} \mathrm{D}_{3}$ & 1305762 & $1349426(-43664)$ \\
\hline 243 & $3 s^{2} 3 p^{5} 4 f$ & ${ }^{3} \mathrm{G}_{4}$ & 1306319 & $1350544(-44225)$ \\
\hline 245 & $3 s^{2} 3 p^{5} 4 f$ & ${ }^{3} \mathrm{G}_{3}$ & 1310158 & $1354494(-44336)$ \\
\hline 246 & $3 s^{2} 3 p^{5} 4 f$ & ${ }^{1} \mathrm{G}_{4}$ & 1311755 & $1356693(-44938)$ \\
\hline 247 & $3 s^{2} 3 p^{5} 4 f$ & ${ }^{3} \mathrm{~F}_{2}$ & 1316758 & $1362088(-45330)$ \\
\hline 250 & $3 s^{2} 3 p^{5} 4 f$ & ${ }^{1} \mathrm{~F}_{3}$ & 1323657 & $1368060(-44403)$ \\
\hline 253 & $3 s^{2} 3 p^{5} 4 f$ & ${ }^{3} \mathrm{~F}_{3}$ & 1324715 & $1369302(-44587)$ \\
\hline 254 & $3 s^{2} 3 p^{5} 4 f$ & ${ }^{3} \mathrm{~F}_{4}$ & 1324876 & $1369536(-44660)$ \\
\hline 256 & $3 s^{2} 3 p^{5} 4 f$ & ${ }^{1} \mathrm{D}_{2}$ & 1331244 & $1376065(-44821)$ \\
\hline 261 & $3 s 3 p^{6} 4 p$ & ${ }^{1} \mathrm{P}_{1}$ & 1371910 & $1397410(-25500)$ \\
\hline 263 & $3 s^{2} 3 p^{5} 5 s$ & ${ }^{1} \mathrm{P}_{1}$ & 1358363 & $1401819(-43456)$ \\
\hline 265 & $3 s^{2} 3 p^{5} 5 s$ & ${ }^{3} \mathrm{P}_{1}$ & 1372683 & $1415509(-42826)$ \\
\hline 302 & $3 s^{2} 3 p^{5} 5 f$ & ${ }^{3} \mathrm{G}_{5}$ & 1513000 & $1567244(-54244)$ \\
\hline 305 & $3 s^{2} 3 p^{5} 5 f$ & ${ }^{3} \mathrm{G}_{4}$ & 1516500 & $1568654(-52154)$ \\
\hline 308 & $3 s^{2} 3 p^{5} 5 f$ & ${ }^{1} \mathrm{~F}_{3}$ & 1519790 & $1570945(-51154)$ \\
\hline 311 & $3 s^{2} 3 p^{5} 5 f$ & ${ }^{3} \mathrm{~F}_{4}$ & 1518650 & $1572181(-53531)$ \\
\hline 316 & $3 s^{2} 3 p^{5} 5 f$ & ${ }^{3} \mathrm{~F}_{2}$ & 1523654 & $1575726(-52072)$ \\
\hline 318 & $3 s^{2} 3 p^{5} 5 f$ & ${ }^{3} \mathrm{~F}_{3}$ & 1531107 & $1584992(-53885)$ \\
\hline 319 & $3 s^{2} 3 p^{5} 5 f$ & ${ }^{1} \mathrm{G}_{4}$ & 1534318 & $1585696(-51377)$ \\
\hline
\end{tabular}

Notes. The columns provide: our experimental level energies $E_{\text {exp }}$ $\left(\mathrm{cm}^{-1}\right)$, those for the 67 configuration target (Table 1) $E_{\text {calc }}\left(\mathrm{cm}^{-1}\right)$. Values in parentheses indicate differences between the observed and theoretical energies. The full dataset is available at the CDS.

$\left(14530 \mathrm{~cm}^{-1}\right)$. For our smaller structure calculations the mixing is: for 27 configurations, $3 \mathrm{~s}^{2} 3 \mathrm{p}^{5} 5 \mathrm{~s}^{1} \mathrm{P}_{1}$ is $52 \%\left(5 \mathrm{~s}{ }^{1} \mathrm{P}_{1}\right)$ plus $45 \%$ $\left(5 \mathrm{~s}{ }^{3} \mathrm{P}_{1}\right)$; for 45 configurations, $3 \mathrm{~s}^{2} 3 \mathrm{p}^{5} 5 \mathrm{~s}{ }^{1} \mathrm{P}_{1}$ is $55 \%\left(5 \mathrm{~s}{ }^{1} \mathrm{P}_{1}\right)$ plus $44 \%\left(5 \mathrm{~s}^{3} \mathrm{P}_{1}\right)$. This change is mainly due to the position of the $3 \mathrm{~s} 3 \mathrm{p}^{6} 4 \mathrm{p}^{1} \mathrm{P}_{1}$ level. Verma et al. quote mixing values for $3 \mathrm{~s}^{2} 3 \mathrm{p}^{5} 5 \mathrm{~s}{ }^{1} \mathrm{P}_{1}$ as $55 \%\left(5 \mathrm{~s}^{1} \mathrm{P}_{1}\right)$ plus $31 \%\left(5 \mathrm{~s}^{3} \mathrm{P}_{1}\right)$, but they also mention that the $3 \mathrm{~s} 3 \mathrm{p}^{6} 4 \mathrm{p}^{1} \mathrm{P}_{1}$ level is coupled with the $3 \mathrm{~s}^{2} 3 \mathrm{p}^{5}$ $5 \mathrm{~s}{ }^{3} \mathrm{P}_{1}$ level, and that they have carried out some semi-empirical adjustments to the Hamiltonian. Our ab-initio model predicts the 
Table 3. Transition probabilities for Fe Ix.

\begin{tabular}{lcccccc}
\hline \hline \multirow{2}{*}{ Levels } & \multicolumn{5}{c}{$A_{j i}\left(\mathrm{~s}^{-1}\right)$} \\
& 27 Conf. & 45 Conf. & 67 Conf. & Storey 02 & Verma 06 & Landi 09 \\
\hline $3 \mathrm{~s}^{2} 3 \mathrm{p}^{6}{ }^{1} \mathrm{~S}_{0}-3 \mathrm{~s}^{2} 3 \mathrm{p}^{5} 4 \mathrm{~s}^{1} \mathrm{P}_{1}$ & $5.40 \times 10^{10}$ & $5.02 \times 10^{10}$ & $5.06 \times 10^{10}$ & $4.1 \times 10^{10}$ & $4.12 \times 10^{10}$ & $4.64 \times 10^{10}$ \\
$3 \mathrm{~s}^{2} 3 \mathrm{p}^{6}{ }^{1} \mathrm{~S}_{0}-3 \mathrm{~s}^{2} 3 \mathrm{p}^{5} 4 \mathrm{~s}^{3} \mathrm{P}_{1}$ & $2.08 \times 10^{10}$ & $2.35 \times 10^{10}$ & $2.43 \times 10^{10}$ & $1.9 \times 10^{10}$ & $2.34 \times 10^{10}$ & $2.20 \times 10^{10}$ \\
$3 \mathrm{~s}^{2} 3 \mathrm{p}^{6}{ }^{1} \mathrm{~S}_{0}-3 \mathrm{~s}^{2} 3 \mathrm{p}^{5} 5 \mathrm{~s}{ }^{1} \mathrm{P}_{1}$ & $1.69 \times 10^{10}$ & $2.66 \times 10^{10}$ & $4.24 \times 10^{10}$ & & $8.98 \times 10^{9}$ & \\
$3 \mathrm{~s}^{2} 3 \mathrm{p}^{6}{ }^{1} \mathrm{~S}_{0}-3 \mathrm{~s}^{2} 3 \mathrm{p}^{5} 5 \mathrm{~s}^{3} \mathrm{P}_{1}$ & $1.40 \times 10^{10}$ & $1.50 \times 10^{10}$ & $1.85 \times 10^{10}$ & & $3.98 \times 10^{10}$ & \\
$3 \mathrm{~s}^{2} 3 \mathrm{p}^{6}{ }^{1} \mathrm{~S}_{0}-3 \mathrm{~s}^{2} 3 \mathrm{p}^{5} 6 \mathrm{~s}^{1} \mathrm{P}_{1}$ & $8.80 \times 10^{9}$ & $1.14 \times 10^{10}$ & $1.06 \times 10^{10}$ & & & \\
$3 \mathrm{~s}^{2} 3 \mathrm{p}^{6}{ }^{1} \mathrm{~S}_{0}-3 \mathrm{~s}^{2} 3 \mathrm{p}^{5} 6 \mathrm{~s}^{3} \mathrm{P}_{1}$ & $4.71 \times 10^{9}$ & $6.30 \times 10^{9}$ & $4.79 \times 10^{9}$ & & & \\
\hline
\end{tabular}

decay from the $5 \mathrm{~s}{ }^{1} \mathrm{P}_{1}$ level to be about twice as strong the decay from the $5 \mathrm{~s}{ }^{3} \mathrm{P}_{1}$. Both Alexander et al. (1965) and Lepson et al. (2002) indicate a ratio of two between these two lines, in agreement with our prediction. We cannot understand why the transition probability given by Verma et al. (their Table 5, our Table 3) for the transition $5 \mathrm{~s}^{1} \mathrm{P}_{1}$ to the ground level is signifcantly less than that for $5 \mathrm{~s}{ }^{3} \mathrm{P}_{1}$ to the ground level. This seems most unusual, even if the mixing with the $3 \mathrm{~s} 3 p^{6} 4{ }^{1} \mathrm{P}_{1}$ level is more significant than we have calculated.

For our largest structure calculation, 67 configurations, the level $3 \mathrm{~s}^{2} 3 \mathrm{p}^{5} 4 \mathrm{~s}^{1} \mathrm{P}_{1}$ is composed of $66 \%\left(4 \mathrm{~s}^{1} \mathrm{P}_{1}\right)$ plus $30 \%$ $\left(4 \mathrm{~s}^{3} \mathrm{P}_{1}\right)$. These values are close to those of Verma, 61\% $\left(4 \mathrm{~s}^{1} \mathrm{P}_{1}\right)$ plus $36 \%\left(4 \mathrm{~s}^{3} \mathrm{P}_{1}\right)$. Our transition probabilities for $4 \mathrm{~s}$ transitions to the ground (Table 3 ) are in reasonable agreement with Verma et al., in contrast to those for $5 \mathrm{~s}$ to the ground.

An R-matrix calculation including all of the excited levels for $n=5 \& 6$ would be quite demanding. We have instead used the distorted wave AUTOSTRUCTURE code to calculate collision strengths for the high lying levels of Fe Ix. These calculations do not include low-energy resonance effects, which could be significant for some transitions. As a result we use the collision strengths from the R-matrix calculation of Storey et al. (2002) for lower levels where available.

Levels up to level 79 contain some metastable levels. For the 79 energetically lowest levels collision strengths are calculated between themselves and up to all excited states. Collisional transitions between excited states higher than level 79 are omitted. Radiative data is calculated for all transitions.

Table 4 contains predicted intensities for the strongest Fe Ix lines in the 50-170 $\AA$ range. The relative intensities (photons) Int $=N_{j} A_{j i} / N_{\mathrm{e}}$ are normalised to the strongest transition and were calculated at electron densities of $10^{8}$ and $10^{12} \mathrm{~cm}^{-3}$ and $\log T[\mathrm{~K}]=5.85$, typical of the quiet solar corona, and of high-density laboratory plasmas. Weighted oscillator strengths $g f$ and $A$-values $\left(\mathrm{s}^{-1}\right)$ are shown. Also included are the calculated and, where available, experimental wavelengths for these lines. The strongest lines are the decays from the $3 \mathrm{~s}^{2} 3 \mathrm{p}^{5} 4 \mathrm{~s}$, d to the ground state, and the $3 \mathrm{~d}-4 \mathrm{f}$ transitions.

One issue of concern is the reliability of DW results for transitions to the $n=4,5$ levels. A comparison between the R-matrix and DW results for the $3 \mathrm{p}-4 \mathrm{~s}$ transitions shows that resonances attached to higher-lying levels are significant. This is caused by the the fact that the $3 s^{2} 3 p^{5} 4$ s levels are relatively low-lying, and the background collision strength is small, hence the contribution from resonances can have an important effect.

For example, the collision strength for the $3 \mathrm{~s}^{2} 3 \mathrm{p}^{6}{ }^{1} \mathrm{~S}_{0}-3 \mathrm{~s}^{2}$ $3 \mathrm{p}^{5} 4 \mathrm{~s}^{1} \mathrm{P}_{1}$ transition is shown in Fig. 1 (top). Good agreement in the background values is found with the R-matrix results from Storey et al. (2002), however the large contributions from the resonances near threshold do affect the thermally-averaged collision strength (shown in Fig. 1, below), with a significant increase
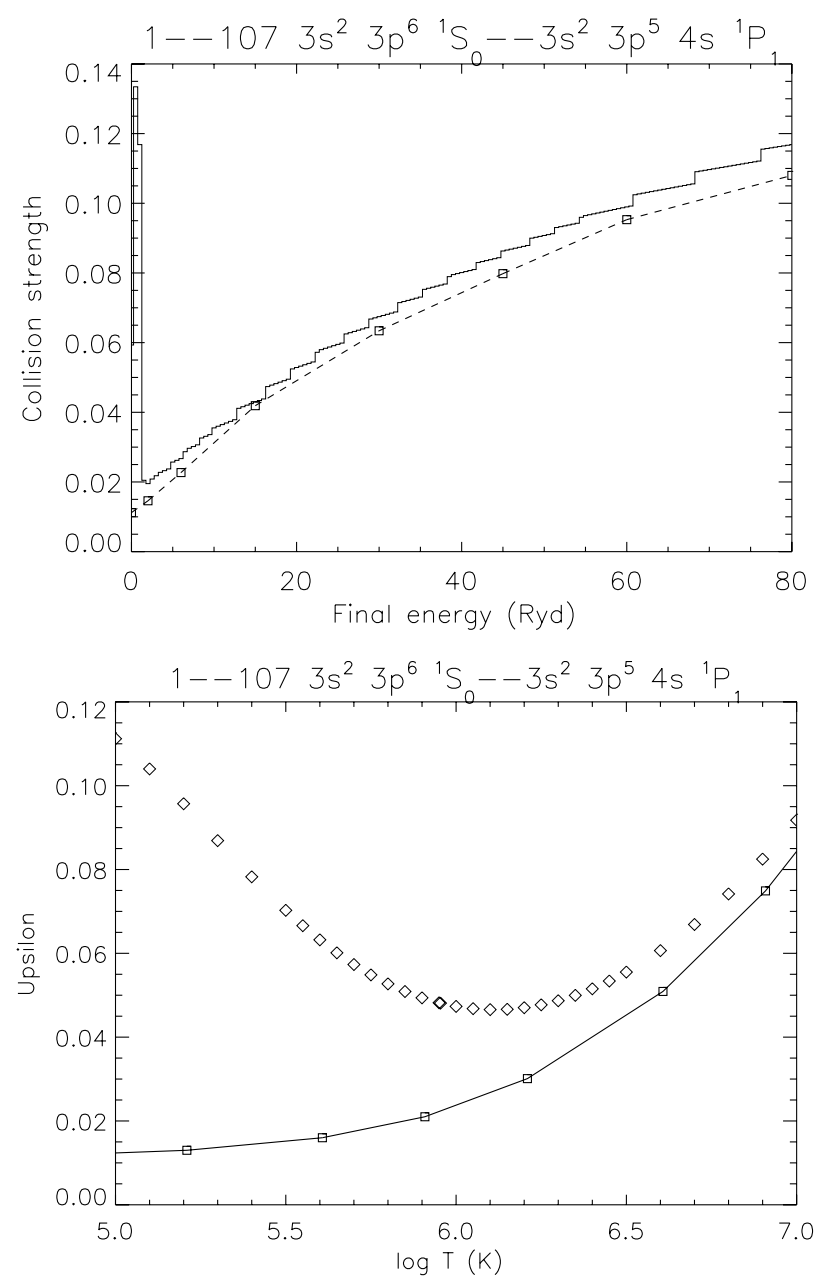

Fig. 1. Top: collision strength for the $3 \mathrm{~s}^{2} 3 \mathrm{p}^{6}{ }^{1} \mathrm{~S}_{0}-3 \mathrm{~s}^{2} 3 \mathrm{p}^{5} 4 \mathrm{~s}^{1} \mathrm{P}_{1}$ transition of Fe IX. Squares with dashed line from present results. Histogram from Storey et al. (2002). Bottom: thermally averaged collision strength for the $3 \mathrm{~s}^{2} 3 \mathrm{p}^{6}{ }^{1} \mathrm{~S}_{0}-3 \mathrm{~s}^{2} 3 \mathrm{p}^{5} 4 \mathrm{~s}^{1} \mathrm{P}_{1}$ transition. Solid line with squares from present results. Diamonds from Storey et al. (2002).

(by a factor of two near peak Fe Ix abundance in equilibrium). Further investigation indicates that the main contribution comes from the $3 \mathrm{~s}^{2} 3 \mathrm{p}^{5} 4 \mathrm{p}^{1} \mathrm{~S}_{0}$ level.

Figure 2 shows the collision strength for the strong $3 s^{2} 3 p^{6}$ ${ }^{1} \mathrm{~S}_{0}-3 \mathrm{~s}^{2} 3 \mathrm{p}^{5} 4 \mathrm{p}{ }^{1} \mathrm{~S}_{0}$ transition. Again, good agreement in the background values is found, as expected. The R-matrix result does not contain resonances because the $3 s^{2} 3 p^{5} 4 p{ }^{1} S_{0}$ level was the highest one included in the Storey et al. (2002) calculation. It is interesting to note the large collision strength for this forbidden transition between configurations with the same parity. This was a somewhat unexpected result. The $3 s^{2} 3 p^{5} 4 p{ }^{1} S_{0}$ 
Table 4. List of the strongest Fe Ix lines in the 50-130 A range.

\begin{tabular}{|c|c|c|c|c|c|c|c|}
\hline$i-j$ & Levels & $\begin{array}{l}\text { Int } \\
10^{8}\end{array}$ & $\begin{array}{c}\text { Int } \\
10^{12}\end{array}$ & $g f$ & $A_{j i}\left(\mathrm{~s}^{-1}\right)$ & $\lambda_{\exp }(\AA)$ & $\lambda_{\mathrm{th}}(\AA)$ \\
\hline $1-107$ & $3 s^{2} 3 p^{6}{ }^{1} S_{0}-3 p^{5} 4 s^{1} P_{1}$ & 1.0 & 0.76 & 0.18 & $4.1 \times 10^{10}$ & 103.566 & $98.08(-5.5)$ \\
\hline $5-239$ & $3 p^{5} 3 d^{3} F_{4}-3 p^{5} 4 f^{3} G_{5}$ & 0.53 & 0.45 & 5.35 & $2.7 \times 10^{11}$ & 113.793 & $108.37(-5.4)$ \\
\hline $1-97$ & $3 \mathrm{~s}^{2} 3 \mathrm{p}^{6}{ }^{1} \mathrm{~S}_{0}-3 \mathrm{p}^{5} 4 \mathrm{~s}^{3} \mathrm{P}_{1}$ & 0.52 & 0.44 & $8.3 \times 10^{-2}$ & $1.9 \times 10^{10}$ & 105.208 & $99.60(-5.6)$ \\
\hline $13-256$ & $3 p^{5} 3 d^{1} P_{1}-3 p^{5} 4 f^{1} D_{2}$ & 0.22 & 0.14 & 1.87 & $1.5 \times 10^{11}$ & 133.923 & $126.34(-7.6)$ \\
\hline $6-243$ & $3 p^{5} 3 d^{3} F_{3}-3 p^{5} 4 f^{3} G_{4}$ & 0.22 & 0.27 & 3.55 & $2.2 \times 10^{11}$ & 114.024 & $108.55(-5.5)$ \\
\hline $1-196$ & $3 \mathrm{~s}^{2} 3 \mathrm{p}^{6}{ }^{1} \mathrm{~S}_{0}-3 \mathrm{p}^{5} 4 \mathrm{~d}^{1} \mathrm{P}_{1}$ & 0.20 & 0.13 & 0.36 & $1.2 \times 10^{11}$ & 83.457 & $80.64(-2.8)$ \\
\hline $1-210$ & $3 s^{2} 3 p^{6}{ }^{1} S_{0}-3 p^{5} 4 d^{3} D_{1}$ & 0.15 & $9.9 \times 10^{-2}$ & 0.12 & $4.3 \times 10^{10}$ & 82.430 & $79.68(-2.7)$ \\
\hline $5-302$ & $3 p^{5} 3 d^{3} F_{4}-3 p^{5} 5 f^{3} G_{5}$ & 0.14 & 0.12 & 2.07 & $1.6 \times 10^{11}$ & 91.980 & $87.61(-4.4)$ \\
\hline $10-247$ & $3 p^{5} 3 d^{3} D_{1}-3 p^{5} 4 f^{3} F_{2}$ & 0.12 & $8.6 \times 10^{-2}$ & 1.29 & $1.4 \times 10^{11}$ & 116.803 & $110.93(-5.9)$ \\
\hline $4-241$ & $3 p^{5} 3 d^{3} P_{2}-3 p^{5} 4 f^{3} D_{3}$ & $9.1 \times 10^{-2}$ & 0.21 & 2.45 & $2.0 \times 10^{11}$ & 112.096 & $106.87(-5.2)$ \\
\hline $10-256$ & $3 p^{5} 3 d^{3} D_{1}-3 p^{5} 4 f^{1} D_{2}$ & $8.9 \times 10^{-2}$ & $5.6 \times 10^{-2}$ & 0.55 & $6.0 \times 10^{10}$ & 114.860 & $109.24(-5.6)$ \\
\hline $8-246$ & $3 p^{5} 3 d^{3} D_{3}-3 p^{5} 4 f^{1} G_{4}$ & $7.9 \times 10^{-2}$ & 0.25 & 3.47 & $2.0 \times 10^{11}$ & 116.803 & $110.98(-5.8)$ \\
\hline $7-245$ & $3 p^{5} 3 d^{3} F_{2}-3 p^{5} 4 f^{3} G_{3}$ & $7.9 \times 10^{-2}$ & 0.16 & 2.37 & $1.9 \times 10^{11}$ & 114.111 & $108.62(-5.5)$ \\
\hline $5-366$ & $3 p^{5} 3 d^{3} F_{4}-3 p^{5} 6 f^{3} G_{5}$ & $7.7 \times 10^{-2}$ & $6.7 \times 10^{-2}$ & 1.64 & $1.6 \times 10^{11}$ & - & 79.12 \\
\hline $3-238$ & $3 p^{5} 3 d^{3} P_{1}-3 p^{5} 4 f^{3} D_{2}$ & $5.7 \times 10^{-2}$ & $7.4 \times 10^{-2}$ & 1.40 & $1.6 \times 10^{11}$ & 111.791 & $106.61(-5.2)$ \\
\hline $13-247$ & $3 p^{5} 3 d^{1} P_{1}-3 p^{5} 4 f^{3} F_{2}$ & $5.7 \times 10^{-2}$ & $4.1 \times 10^{-2}$ & 0.84 & $6.5 \times 10^{10}$ & 136.572 & $128.61(-8.0)$ \\
\hline $13-276$ & $3 p^{5} 3 d^{1} P_{1}-3 p^{5} 5 p^{1} S_{0}$ & $5.6 \times 10^{-2}$ & $3.2 \times 10^{-2}$ & $6.5 \times 10^{-3}$ & $3.2 \times 10^{9}$ & - & 113.08 \\
\hline $10-271$ & $3 p^{5} 3 d^{3} D_{1}-3 p^{5} 5 p^{3} P_{0}$ & $5.4 \times 10^{-2}$ & $3.1 \times 10^{-2}$ & $4.9 \times 10^{-2}$ & $3.1 \times 10^{10}$ & - & 100.76 \\
\hline $6-305$ & $3 p^{5} 3 d^{3} F_{3}-3 p^{5} 5 f^{3} G_{4}$ & $5.2 \times 10^{-2}$ & $6.1 \times 10^{-2}$ & 1.33 & $1.3 \times 10^{11}$ & 91.980 & $87.77(-4.2)$ \\
\hline $12-254$ & $3 p^{5} 3 d^{1} F_{3}-3 p^{5} 4 f^{3} F_{4}$ & $5.0 \times 10^{-2}$ & 0.24 & 3.52 & $2.1 \times 10^{11}$ & 116.408 & $110.66(-5.8)$ \\
\hline $13-326$ & $3 p^{5} 3 d^{1} P_{1}-3 p^{5} 5 f^{1} D_{2}$ & $4.9 \times 10^{-2}$ & $3.3 \times 10^{-2}$ & 0.73 & $9.5 \times 10^{10}$ & - & 99.51 \\
\hline $13-379$ & $3 p^{5} 3 d^{1} P_{1}-3 p^{5}$ of ${ }^{1} D_{2}$ & $4.6 \times 10^{-2}$ & $3.0 \times 10^{-2}$ & 0.50 & $8.4 \times 10^{10}$ & - & 88.30 \\
\hline $10-276$ & $3 p^{5} 3 d^{3} D_{1}-3 p^{5} 5 p^{1} S_{0}$ & $4.6 \times 10^{-2}$ & $2.6 \times 10^{-2}$ & $4.0 \times 10^{-3}$ & $2.7 \times 10^{9}$ & - & 99.18 \\
\hline $10-316$ & $3 p^{5} 3 d^{3} D_{1}-3 p^{5} 5 f^{3} F_{2}$ & $3.5 \times 10^{-2}$ & $2.4 \times 10^{-2}$ & 0.44 & $7.2 \times 10^{10}$ & 94.070 & $89.68(-4.4)$ \\
\hline $11-253$ & $3 p^{5} 3 d^{3} D_{2}-3 p^{5} 4 f^{3} F_{3}$ & $3.4 \times 10^{-2}$ & 0.16 & 2.76 & $2.1 \times 10^{11}$ & 115.996 & $110.29(-5.7)$ \\
\hline $2-237$ & $3 p^{5} 3 d^{3} P_{0}-3 p^{5} 4 f^{3} D_{1}$ & $3.3 \times 10^{-2}$ & $3.5 \times 10^{-2}$ & 0.66 & $1.3 \times 10^{11}$ & 111.713 & $106.55(-5.2)$ \\
\hline $9-250$ & $3 p^{5} 3 d^{1} D_{2}-3 p^{5} 4 f^{1} F_{3}$ & $3.2 \times 10^{-2}$ & 0.15 & 2.42 & $1.9 \times 10^{11}$ & 115.353 & $109.73(-5.6)$ \\
\hline $17-349$ & $3 p^{6} 3 d^{1} D_{2}-3 p^{6} 4 f^{1} F_{3}$ & $2.8 \times 10^{-2}$ & $1.9 \times 10^{-2}$ & 2.34 & $1.7 \times 10^{11}$ & - & 111.34 \\
\hline $3-237$ & $3 p^{5} 3 d^{3} P_{1}-3 p^{5} 4 f^{3} D_{1}$ & $2.7 \times 10^{-2}$ & $2.9 \times 10^{-2}$ & 0.55 & $1.1 \times 10^{11}$ & 112.031 & $106.84(-5.2)$ \\
\hline $6-368$ & $3 p^{5} 3 d^{3} F_{3}-3 p^{5} 6 f^{3} G_{4}$ & $2.6 \times 10^{-2}$ & $3.1 \times 10^{-2}$ & 0.87 & $1.0 \times 10^{11}$ & - & 79.23 \\
\hline $8-311$ & $3 p^{5} 3 d^{3} D_{3}-3 p^{5} 5 f^{3} F_{4}$ & $2.6 \times 10^{-2}$ & $6.9 \times 10^{-2}$ & 1.44 & $1.3 \times 10^{11}$ & 94.070 & $89.56(-4.5)$ \\
\hline $13-316$ & $3 p^{5} 3 d^{1} P_{1}-3 p^{5} 5 f^{3} F_{2}$ & $2.5 \times 10^{-2}$ & $1.7 \times 10^{-2}$ & 0.41 & $5.2 \times 10^{10}$ & - & 100.89 \\
\hline $1-280$ & $3 \mathrm{~s}^{2} 3 \mathrm{p}^{6}{ }^{1} \mathrm{~S}_{0}-3 \mathrm{p}^{5} 5 \mathrm{~d}^{1} \mathrm{P}_{1}$ & $2.5 \times 10^{-2}$ & $1.6 \times 10^{-2}$ & 0.16 & $7.8 \times 10^{10}$ & - & 66.39 \\
\hline $11-256$ & $3 p^{5} 3 d^{3} D_{2}-3 p^{5} 4 f^{1} D_{2}$ & $2.5 \times 10^{-2}$ & $1.6 \times 10^{-2}$ & 0.16 & $1.7 \times 10^{10}$ & 115.124 & $109.48(-5.6)$ \\
\hline $4-304$ & $3 p^{5} 3 d^{3} P_{2}-3 p^{5} 5 f^{3} D_{3}$ & $2.4 \times 10^{-2}$ & $4.3 \times 10^{-2}$ & 0.81 & $1.0 \times 10^{11}$ & - & 86.59 \\
\hline $4-238$ & $3 p^{5} 3 d^{3} P_{2}-3 p^{5} 4 f^{3} D_{2}$ & $2.4 \times 10^{-2}$ & $3.1 \times 10^{-2}$ & 0.59 & $6.7 \times 10^{10}$ & 112.464 & $107.23(-5.2)$ \\
\hline $7-247$ & $3 p^{5} 3 d^{3} F_{2}-3 p^{5} 4 f^{3} F_{2}$ & $2.3 \times 10^{-2}$ & $1.7 \times 10^{-2}$ & 0.24 & $2.6 \times 10^{10}$ & 113.258 & $107.73(-5.5)$ \\
\hline $10-326$ & $3 p^{5} 3 d^{3} D_{1}-3 p^{5} 5 f^{1} D_{2}$ & $2.2 \times 10^{-2}$ & $1.5 \times 10^{-2}$ & 0.26 & $4.3 \times 10^{10}$ & - & 88.58 \\
\hline $8-241$ & $3 p^{5} 3 d^{3} D_{3}-3 p^{5} 4 f^{3} D_{3}$ & $2.1 \times 10^{-2}$ & $4.9 \times 10^{-2}$ & 0.63 & $4.7 \times 10^{10}$ & 117.626 & $111.88(-5.7)$ \\
\hline $8-370$ & $3 p^{5} 3 d^{3} D_{3}-3 p^{5} 6 f^{3} F_{4}$ & $2.0 \times 10^{-2}$ & $4.5 \times 10^{-2}$ & 1.32 & $1.5 \times 10^{11}$ & - & 80.61 \\
\hline $12-243$ & $3 p^{5} 3 d^{1} F_{3}-3 p^{5} 4 f^{3} G_{4}$ & $2.0 \times 10^{-2}$ & $2.4 \times 10^{-2}$ & 0.34 & $2.0 \times 10^{10}$ & 118.978 & $113.03(-5.9)$ \\
\hline $1-263$ & $3 \mathrm{~s}^{2} 3 \mathrm{p}^{6}{ }^{1} \mathrm{~S}_{0}-3 \mathrm{p}^{5} 5 \mathrm{~s}^{1} \mathrm{P}_{1}$ & $1.9 \times 10^{-2}$ & $1.2 \times 10^{-2}$ & $9.7 \times 10^{-2}$ & $4.2 \times 10^{10}$ & 73.618 & $71.34(-2.3)$ \\
\hline 13-339 & $3 p^{5} 3 d^{1} P_{1}-3 p^{5} 6 p^{1} S_{0}$ & $1.9 \times 10^{-2}$ & $1.1 \times 10^{-2}$ & $4.6 \times 10^{-3}$ & $3.3 \times 10^{9}$ & - & 95.48 \\
\hline $3-301$ & $3 p^{5} 3 d^{3} P_{1}-3 p^{5} 5 f^{3} D_{2}$ & $1.9 \times 10^{-2}$ & $2.0 \times 10^{-2}$ & 0.50 & $8.9 \times 10^{10}$ & - & 86.34 \\
\hline $5-243$ & $3 p^{5} 3 d^{3} F_{4}-3 p^{5} 4 f^{3} G_{4}$ & $1.9 \times 10^{-2}$ & $2.3 \times 10^{-2}$ & 0.30 & $1.9 \times 10^{10}$ & 113.571 & $108.14(-5.4)$ \\
\hline $12-319$ & $3 p^{5} 3 d^{1} F_{3}-3 p^{5} 5 f^{1} G_{4}$ & $1.8 \times 10^{-2}$ & $5.8 \times 10^{-2}$ & 1.40 & $1.3 \times 10^{11}$ & 93.590 & $89.30(-4.3)$ \\
\hline $7-308$ & $3 p^{5} 3 d^{3} F_{2}-3 p^{5} 5 f^{1} F_{3}$ & $1.7 \times 10^{-2}$ & $3.0 \times 10^{-2}$ & 0.73 & $8.9 \times 10^{10}$ & 91.980 & $87.94(-4.1)$ \\
\hline $11-318$ & $3 p^{5} 3 d^{3} D_{2}-3 p^{5} 5 f^{3} F_{3}$ & $1.6 \times 10^{-2}$ & $4.3 \times 10^{-2}$ & 0.96 & $1.1 \times 10^{11}$ & 93.590 & $89.10(-4.5)$ \\
\hline $1-265$ & $3 \mathrm{~s}^{2} 3 \mathrm{p}^{6}{ }^{1} \mathrm{~S}_{0}-3 \mathrm{p}^{5} 5 \mathrm{~s}^{3} \mathrm{P}_{1}$ & $1.1 \times 10^{-2}$ & $7.3 \times 10^{-3}$ & $4.2 \times 10^{-2}$ & $1.8 \times 10^{10}$ & 72.850 & $70.65(-2.2)$ \\
\hline 9-308 & $3 p^{5} 3 d^{1} D_{2}-3 p^{5} 5 f^{1} F_{3}$ & $7.1 \times 10^{-3}$ & $1.3 \times 10^{-2}$ & 0.32 & $3.7 \times 10^{10}$ & 94.070 & $89.75(-4.3)$ \\
\hline
\end{tabular}

Notes. The relative intensities (photons) Int $=N_{j} A_{j i} / N_{\mathrm{e}}$ are normalised to the strongest transition and were calculated at electron densities of $10^{8}$ and $10^{12} \mathrm{~cm}^{-3}$ and $\log T[\mathrm{~K}]=5.85$. Weighted oscillator strengths $g f$ and $A$-values $\left(\mathrm{s}^{-1}\right)$ are shown. Experimental $\lambda_{\exp }(\AA)$ and theoretical $\lambda_{\text {th }}(\AA)$ wavelengths are included. Values in parentheses indicate differences between the observed and theoretical wavelengths.

level decays via a strong dipole-allowed transition to the $3 \mathrm{~s}^{2} 3 \mathrm{p}^{5}$ $3 \mathrm{~d}^{1} \mathrm{P}_{1}$. An estimate of the wavelength for this transition with our 67 configuration calculation provides a value very close to that suggested by Young (2009), who identified this transition with a line in the Hinode EIS spectrum, at $197.862 \AA$. The observed intensity of this line, relative to that of other Fe IX transitions, is in good agreement with that predicted using the Storey et al. (2002) calculation, hence indirectly confirming the large collision strength from the ground state to the $3 s^{2} 3 p^{5} 4 p^{1} S_{0}$ level.

We have run various checks by looking at the collision strengths near thresholds for all transitions to levels with $n \geq 4$, and we believe that significant enhancements due to resonances 

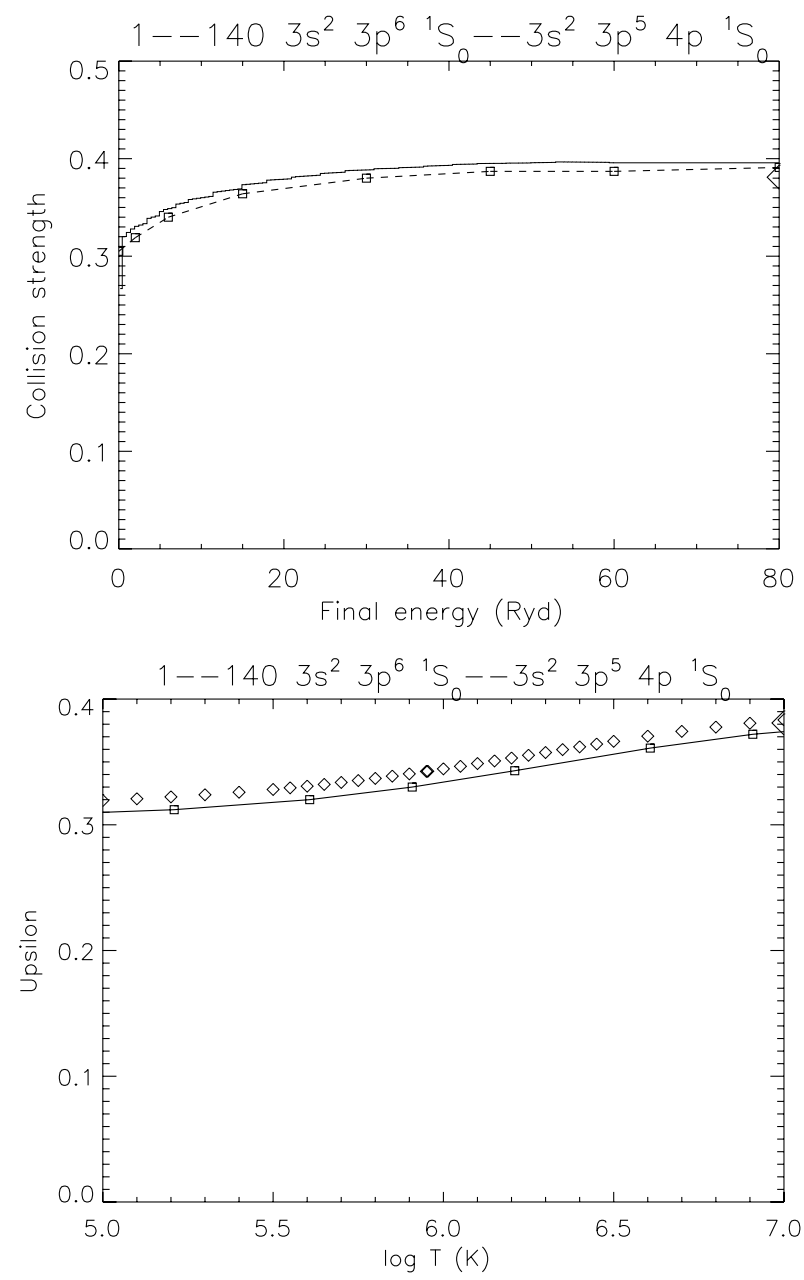

Fig. 2. Top: collision strength for the $3 s^{2} 3 p^{6}{ }^{1} S_{0}-3 s^{2} 3 p^{5} 4 p{ }^{1} S_{0}$ transition of Fe IX. Squares with dashed line from present results. Histogram from Storey et al. (2002). Bottom: thermally averaged collision strength for the $3 s^{2} 3 p^{6}{ }^{1} S_{0}-3 s^{2} 3 p^{5} 4 p{ }^{1} S_{0}$ transition. Solid line with squares from present results. Diamonds from Storey et al. (2002).

such as those occurring to the $3 s^{2} 3 p^{5} 4$ s levels should not be present. However, a further in-depth analysis, which is beyond the scope of this paper, is in progress.

\section{Fe VIII}

The identification of Fe vIII lines is reviewed in Del Zanna (2009). It started with Kruger \& Weissberg (1937), who identified the main decays of the 4f, 5f, $6 \mathrm{f}$ and $7 \mathrm{f}$ levels. That study was followed by the fundamental laboratory work of Fawcett and co-workers on the ZETA spectrum (see Gabriel \& Fawcett 1965), where a number of identifications for the EUV lines were provided. The main source of accurate wavelengths and further identifications came from the excellent work of Ramonas \& Ryabtsev (1980).

Several atomic structure calculations exist in the literature. The latest ones are from Zeng et al. (2003), and Del Zanna (2009). Zeng et al. (2003) performed large-scale multi-configuration Hartree-Fock (MCHF) calculations for Fe vIII, to show the importance of including core-valence electron correlations. Del Zanna (2009) used Autostructure and searched a "benchmark" configuration basis which reproduced well the positioning of a few key low $n=3,4$ mixed levels.
Table 5. Electron configuration basis for the DW calculation and orbital scaling parameters for Fe viII.

\begin{tabular}{lccc}
\hline \hline \multicolumn{3}{c}{ Configurations } & \multicolumn{2}{l}{ Scaling parameters } \\
even & odd & & \\
\hline $3 \mathrm{~s}^{2} 3 \mathrm{p}^{6} 3 \mathrm{~d}$ & $3 \mathrm{~s}^{2} 3 \mathrm{p}^{5} 3 \mathrm{~d}^{2}$ & $1 \mathrm{~s}: 1.4209$ & $2 \mathrm{~s}: 1.1289$ \\
$3 \mathrm{~s}^{2} 3 \mathrm{p}^{6} 4 \mathrm{~s}$ & $3 \mathrm{~s}^{2} 3 \mathrm{p}^{6} 4 \mathrm{p}$ & $2 \mathrm{p}: 1.0689$ & $3 \mathrm{~s}: 1.1503$ \\
$3 \mathrm{~s}^{2} 3 \mathrm{p}^{6} 4 \mathrm{~d}$ & $3 \mathrm{~s}^{2} 3 \mathrm{p}^{6} 4 \mathrm{f}$ & $3 \mathrm{p}: 1.1315$ & $3 \mathrm{~d}: 1.1576$ \\
$3 \mathrm{~s}^{2} 3 \mathrm{p}^{6} 5 \mathrm{~s}$ & $3 \mathrm{~s}^{2} 3 \mathrm{p}^{6} 5 \mathrm{p}$ & $4 \mathrm{~s}: 1.1520$ & $4 \mathrm{p}: 1.1194$ \\
$3 \mathrm{~s}^{2} 3 \mathrm{p}^{6} 5 \mathrm{~d}$ & $3 \mathrm{~s}^{2} 3 \mathrm{p}^{6} 5 \mathrm{f}$ & $4 \mathrm{~d}: 1.1183$ & $4 \mathrm{f}: 1.2042$ \\
$3 \mathrm{~s}^{2} 3 \mathrm{p}^{6} 5 \mathrm{~g}$ & $3 \mathrm{~s}^{2} 3 \mathrm{p}^{6} 6 \mathrm{p}$ & $5 \mathrm{~s}: 1.1468$ & $5 \mathrm{p}: 1.1229$ \\
$3 \mathrm{~s}^{2} 3 \mathrm{p}^{6} 6 \mathrm{~s}$ & $3 \mathrm{~s}^{2} 3 \mathrm{p}^{6} 6 \mathrm{f}$ & $5 \mathrm{~d}: 1.1224$ & $5 \mathrm{f}: 1.2009$ \\
$3 \mathrm{~s}^{2} 3 \mathrm{p}^{6} 6 \mathrm{~d}$ & $3 \mathrm{~s}^{2} 3 \mathrm{p}^{6} 6 \mathrm{~h}$ & $5 \mathrm{~g}: 1.2170$ & $6 \mathrm{~s}: 1.1489$ \\
$3 \mathrm{~s}^{2} 3 \mathrm{p}^{6} 6 \mathrm{~g}$ & $3 \mathrm{~s}^{2} 3 \mathrm{p}^{6} 7 \mathrm{p}$ & $6 \mathrm{p}: 1.1257$ & $6 \mathrm{~d}: 1.1250$ \\
$3 \mathrm{~s}^{2} 3 \mathrm{p}^{6} 7 \mathrm{~s}$ & $3 \mathrm{~s}^{2} 3 \mathrm{p}^{6} 7 \mathrm{f}$ & $6 \mathrm{f}: 1.2004$ & $6 \mathrm{~g}: 1.2184$ \\
$3 \mathrm{~s}^{2} 3 \mathrm{p}^{6} 7 \mathrm{~d}$ & $3 \mathrm{~s}^{2} 3 \mathrm{p}^{5} 3 \mathrm{~d} 4 \mathrm{~s}$ & $6 \mathrm{~h}: 1.2174$ & $7 \mathrm{~s}: 1.1518$ \\
------------- & & \\
$3 \mathrm{~s}^{2} 3 \mathrm{p}^{4} 3 \mathrm{~d}^{2} 4 \mathrm{f}$ & & $7 \mathrm{p}: 1.1291$ & $7 \mathrm{~d}: 1.1275$ \\
\hline
\end{tabular}

Notes. The configuration below the dashed line is included for correlation purposes.

The additional $n>4$ configurations were added to obtain a good target for these levels.

The latest electron scattering calculation for this ion is the R-matrix calculation by Griffin et al. (2000). It included the 33 terms and the 77 levels of the configurations $3 s^{2} 3 p^{6} 3 d$, $3 s^{2} 3 p^{5} 3 d^{2}, 3 s^{2} 3 p^{5} 3 d 4 s, 3 s^{2} 3 p^{6} 4 s, 3 s^{2} 3 p^{6} 4 p, 3 s^{2} 3 p^{6} 4 d$ and $3 s^{2} 3 p^{6}$ 4f in the close-coupling expansion. The Griffin et al. (2000) data have been benchmarked by Del Zanna (2009) using Hinode EUV Imaging Spectrometer (EIS, see Culhane et al. 2007) observations, measurements found in the literature, and laboratory plates from B.C. Fawcett (see Del Zanna et al. 2004, for details on the benchmark method). The benchmark work showed that significant discrepancies ( $60 \%$ or so) between Hinode EIS observed intensities and those predicted with the Griffin et al. (2000) data exist for some lines. It was shown with the run of a large-scale AUTOSTRUCTURE "benchmark" calculation that this was due to missing configuration interaction (CI). The effects are subtle for some strong transitions arising from very mixed levels, and were also noted by Griffin et al. (2000).

In order to improve the Griffin et al. (2000) data, the collision strengths of the dipole-allowed transitions were scaled by Del Zanna (2009) according to the ratio of the gf values (and energies) in the Griffin et al. (2000) and the "benchmark" calculation. These data are included in CHIANTI v.7 and are adopted here.

The structure calculation by Del Zanna et al. (2004) was optimised for transitions between the $n=3$ configurations. We have extended the structure (and scattering) calculations to levels up to $n=7$.

An R-matrix calculation including all of the excited levels for $n=5,6 \& 7$ would be quite demanding. Instead we use the distorted wave AUTOSTRUCTURE code to calculate collision strengths between levels of Fe viII included in our structure calculations. Levels up to level 40 contain some metastable levels. For the 40 energetically lowest levels collision strengths are calculated between themselves and to all excited states. Collisional transitions between excited states higher than level 40 are omitted. Radiative data is calculated for all transitions.

The target wavefunctions are expanded in the 23 configuration basis listed in Table 5. The configuration $3 \mathrm{~s}^{2} 3 \mathrm{p}^{4} 3 \mathrm{~d}^{2} 4 \mathrm{f}$ is 


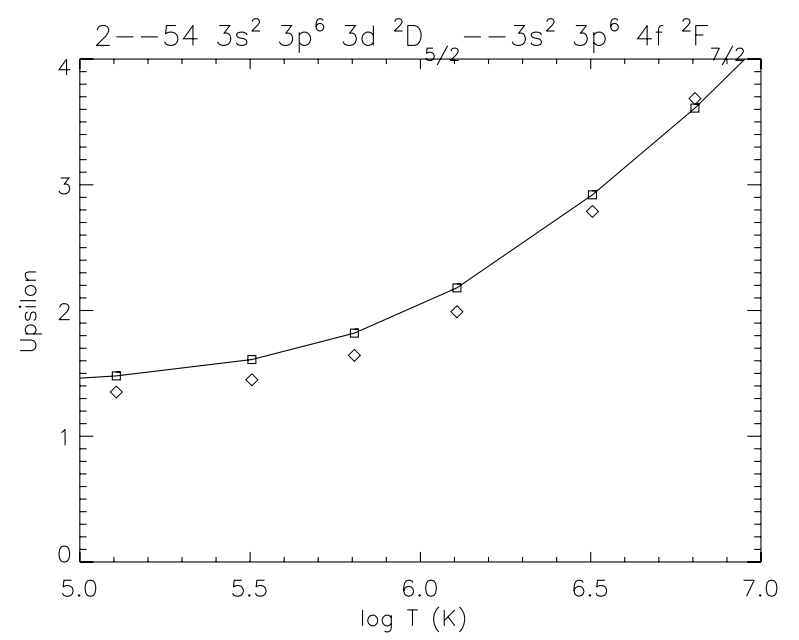

Fig. 3. Thermally averaged collision strength for the $3 \mathrm{~s}^{2} 3 \mathrm{p}^{6} 3 \mathrm{~d}^{2} \mathrm{D}_{5 / 2}-$ $3 \mathrm{~s}^{2} 3 \mathrm{p}^{6} 4 \mathrm{f}^{2} \mathrm{~F}_{7 / 2}$ transition of $\mathrm{Fe}$ viII. Solid line from present results. Diamonds from the R-matrix calculation of Griffin et al. (2000), adjusted by Del Zanna (2009).

present solely for correlation purposes. The resulting scaling parameters are also given in Table 5.

In Table 6 we list the calculated and, where available, experimental energies for a sample of levels of the target. A complete list of the calculated energies of all 104 levels of the target is available at the CDS.

Table 7 contains predicted intensities for the strongest Fe vIII lines in the 50-170 $\AA$ range. The relative intensities (photons) Int $=N_{j} A_{j i} / N_{\mathrm{e}}$ are normalised to the strongest transition and were calculated at electron densities of $10^{8}$ and $10^{12} \mathrm{~cm}^{-3}$ and $\log T[\mathrm{~K}]=5$.6. Weighted oscillator strengths $g f$ and $A$-values $\left(\mathrm{s}^{-1}\right)$ are shown. Aslo included are the calculated and, where available, experimental wavelengths for these lines.

Thermally averaged collision strength for the $3 s^{2} 3 p^{6} 3 d$ ${ }^{2} \mathrm{D}_{5 / 2}-3 \mathrm{~s}^{2} 3 \mathrm{p}^{6} 4 \mathrm{f}^{2} \mathrm{~F}_{7 / 2}$ transition of Fe vIII is plotted as a function of temperature in Fig. 3. The corresponding thermally averaged collision strength from the R-matrix calculation of Griffin et al. (2000), adjusted by Del Zanna, is found to be in very good agreement.

\section{Benchmarking the atomic data}

There is relatively little experimental data that we can use to benchmark the atomic data we have calculated. Most solar spectra have a relatively low resolution or are not calibrated. In terms of spectral resolution, the best soft X-ray solar spectrum is from Behring et al. (1972). They published a line list based on a LASP rocket flight that observed the entire Sun in the 60-385 Å region with very high-resolution $(0.06 \AA)$. A list of those lines observed in quiet conditions are included in Table 8 . In terms of both spectral resolution and flux calibration, the best soft X-ray spectra of the quiet Sun are from Manson (1972). Manson (1972) provided an excellent list of lines observed in quiet and active conditions. The list of lines observed in quiet conditions are included in Table 8.

In terms of laboratory spectra, we use here the flux-calibrated theta-pinch spectra of Datla et al. (1975), and the EBIT measurements of Lepson et al. (2002). Lepson et al. provided some estimates of the line intensities. We are not sure about the actual flux calibration, so only lines not too far in wavelength are considered here.
Table 6. Level energies for Fe viII.

\begin{tabular}{|c|c|c|c|c|}
\hline$i$ & Conf. & Lev. & $E_{\exp }$ & $E_{\text {calc }}$ \\
\hline 1 & $3 s^{2} 3 p^{6} 3 d$ & ${ }^{2} \mathrm{D}_{3 / 2}$ & 0.0 & 0.0 \\
\hline 2 & $3 s^{2} 3 p^{6} 3 d$ & ${ }^{2} \mathrm{D}_{5 / 2}$ & 1836 & $2038(-202)$ \\
\hline 3 & $3 s^{2} 3 p^{5} 3 d^{2}$ & ${ }^{4} \mathrm{D}_{1 / 2}$ & 391108 & 384967 (6141) \\
\hline 4 & $3 s^{2} 3 p^{5} 3 d^{2}$ & ${ }^{4} \mathrm{D}_{3 / 2}$ & 391988 & 385994 (5994) \\
\hline 5 & $3 s^{2} 3 p^{5} 3 d^{2}$ & ${ }^{4} \mathrm{D}_{5 / 2}$ & 393455 & $387729(5726)$ \\
\hline 6 & $3 s^{2} 3 p^{5} 3 d^{2}$ & ${ }^{4} \mathrm{D}_{7 / 2}$ & 395605 & 390297 (5308) \\
\hline 7 & $3 s^{2} 3 p^{5} 3 d^{2}$ & ${ }^{4} \mathrm{G}_{11 / 2}$ & - & 408204 \\
\hline 8 & $3 s^{2} 3 p^{5} 3 d^{2}$ & ${ }^{4} \mathrm{G}_{9 / 2}$ & - & 409793 \\
\hline 9 & $3 s^{2} 3 p^{5} 3 d^{2}$ & ${ }^{4} \mathrm{G}_{7 / 2}$ & - & 411883 \\
\hline 10 & $3 s^{2} 3 p^{5} 3 d^{2}$ & ${ }^{4} \mathrm{P}_{5 / 2}$ & - & 413481 \\
\hline 11 & $3 s^{2} 3 p^{5} 3 d^{2}$ & ${ }^{4} \mathrm{G}_{5 / 2}$ & - & 414197 \\
\hline 12 & $3 s^{2} 3 p^{5} 3 d^{2}$ & ${ }^{4} \mathrm{P}_{3 / 2}$ & - & 416763 \\
\hline 13 & $3 s^{2} 3 p^{5} 3 d^{2}$ & ${ }^{4} \mathrm{P}_{1 / 2}$ & - & 419214 \\
\hline 14 & $3 s^{2} 3 p^{5} 3 d^{2}$ & ${ }^{4} \mathrm{~F}_{3 / 2}$ & - & 423625 \\
\hline 15 & $3 s^{2} 3 p^{6} 4 s$ & ${ }^{2} \mathrm{~S}_{1 / 2}$ & - & 424077 \\
\hline 41 & $3 s^{2} 3 p^{6} 4 p$ & ${ }^{2} \mathrm{P}_{1 / 2}$ & 510277 & $509420(857)$ \\
\hline 42 & $3 s^{2} 3 p^{6} 4 p$ & ${ }^{2} \mathrm{P}_{3 / 2}$ & 515550 & $512194(3356)$ \\
\hline 43 & $3 s^{2} 3 p^{5} 3 d^{2}$ & ${ }^{2} \mathrm{P}_{3 / 2}$ & 508518 & $521315(-12797)$ \\
\hline 44 & $3 s^{2} 3 p^{5} 3 d^{2}$ & ${ }^{2} \mathrm{P}_{1 / 2}$ & 520822 & $531502(-10680)$ \\
\hline 45 & $3 s^{2} 3 p^{5} 3 d^{2}$ & ${ }^{2} \mathrm{~F}_{5 / 2}$ & 535910 & $553736(-17826)$ \\
\hline 46 & $3 s^{2} 3 p^{5} 3 d^{2}$ & ${ }^{2} \mathrm{~F}_{7 / 2}$ & 541755 & $559876(-18121)$ \\
\hline 47 & $3 s^{2} 3 p^{5} 3 d^{2}$ & ${ }^{2} \mathrm{P}_{1 / 2}$ & 591964 & $611822(-19858)$ \\
\hline 48 & $3 s^{2} 3 p^{5} 3 d^{2}$ & ${ }^{2} \mathrm{P}_{3 / 2}$ & 595152 & $614976(-19824)$ \\
\hline 49 & $3 s^{2} 3 p^{5} 3 d^{2}$ & ${ }^{2} \mathrm{D}_{5 / 2}$ & 596465 & $624968(-28503)$ \\
\hline 50 & $3 s^{2} 3 p^{5} 3 d^{2}$ & ${ }^{2} \mathrm{D}_{3 / 2}$ & 597065 & $625143(-28078)$ \\
\hline 51 & $3 s^{2} 3 p^{6} 4 d$ & ${ }^{2} \mathrm{D}_{3 / 2}$ & 653717 & $657978(-4261)$ \\
\hline 52 & $3 s^{2} 3 p^{6} 4 d$ & ${ }^{2} \mathrm{D}_{5 / 2}$ & 654197 & $658461(-4264)$ \\
\hline 53 & $3 s^{2} 3 p^{6} 4 f$ & ${ }^{2} \mathrm{~F}_{5 / 2}$ & 763703 & $728932(34771)$ \\
\hline 54 & $3 s^{2} 3 p^{6} 4 f$ & ${ }^{2} \mathrm{~F}_{7 / 2}$ & 763799 & $729150(34649)$ \\
\hline 61 & $3 s^{2} 3 p^{5} 3 d 4 s$ & ${ }^{2} \mathrm{P}_{1 / 2}$ & 837661 & $839279(-1618)$ \\
\hline 62 & $3 s^{2} 3 p^{5} 3 d 4 s$ & ${ }^{4} \mathrm{~F}_{9 / 2}$ & - & 844540 \\
\hline 63 & $3 s^{2} 3 p^{5} 3 d 4 s$ & ${ }^{2} \mathrm{P}_{3 / 2}$ & 842829 & $844766(-1937)$ \\
\hline 64 & $3 s^{2} 3 p^{5} 3 d 4 s$ & ${ }^{4} \mathrm{~F}_{7 / 2}$ & 847145 & $847137(8)$ \\
\hline 65 & $3 s^{2} 3 p^{5} 3 d 4 s$ & ${ }^{4} \mathrm{~F}_{5 / 2}$ & 849899 & $849973(-74)$ \\
\hline 66 & $3 s^{2} 3 p^{5} 3 d 4 s$ & ${ }^{4} \mathrm{~F}_{3 / 2}$ & 852849 & $853003(-154)$ \\
\hline 67 & $3 s^{2} 3 p^{5} 3 d 4 s$ & ${ }^{2} \mathrm{~F}_{7 / 2}$ & 855100 & $856493(-1393)$ \\
\hline 68 & $3 s^{2} 3 p^{5} 3 d 4 s$ & ${ }^{2} \mathrm{~F}_{5 / 2}$ & 860615 & $862322(-1707)$ \\
\hline 69 & $3 s^{2} 3 p^{5} 3 d 4 s$ & ${ }^{4} \mathrm{D}_{7 / 2}$ & 874711 & $878133(-3422)$ \\
\hline 70 & $3 s^{2} 3 p^{6} 5 d$ & ${ }^{2} \mathrm{D}_{3 / 2}$ & - & 878690 \\
\hline 71 & $3 \mathrm{~s}^{2} 3 \mathrm{p}^{6} 5 \mathrm{~d}$ & ${ }^{2} \mathrm{D}_{5 / 2}$ & - & 878916 \\
\hline 72 & $3 s^{2} 3 p^{5} 3 d 4 s$ & ${ }^{4} \mathrm{D}_{5 / 2}$ & 876765 & $880421(-3656)$ \\
\hline 73 & $3 s^{2} 3 p^{5} 3 d 4 s$ & ${ }^{4} \mathrm{D}_{3 / 2}$ & 877476 & $881507(-4031)$ \\
\hline 74 & $3 s^{2} 3 p^{5} 3 d 4 s$ & ${ }^{4} \mathrm{D}_{1 / 2}$ & 878264 & $882329(-4065)$ \\
\hline 75 & $3 s^{2} 3 p^{5} 3 d 4 s$ & ${ }^{2} \mathrm{D}_{5 / 2}$ & 879021 & $884089(-5068)$ \\
\hline 76 & $3 s^{2} 3 p^{5} 3 d 4 s$ & ${ }^{2} \mathrm{D}_{3 / 2}$ & 881345 & $886603(-5258)$ \\
\hline 77 & $3 s^{2} 3 p^{5} 3 d 4 s$ & ${ }^{2} \mathrm{~F}_{5 / 2}$ & 884331 & $888349(-4018)$ \\
\hline 78 & $3 s^{2} 3 p^{5} 3 d 4 s$ & ${ }^{2} \mathrm{~F}_{7 / 2}$ & 887325 & $890584(-3259)$ \\
\hline 79 & $3 s^{2} 3 p^{5} 3 d 4 s$ & ${ }^{2} \mathrm{D}_{3 / 2}$ & 889113 & $893935(-4822)$ \\
\hline 80 & $3 s^{2} 3 p^{5} 3 d 4 s$ & ${ }^{2} \mathrm{D}_{5 / 2}$ & 890845 & $895255(-4410)$ \\
\hline 81 & $3 s^{2} 3 p^{6} 5 f$ & ${ }^{2} \mathrm{~F}_{5 / 2}$ & 927059 & $926655(404)$ \\
\hline 82 & $3 s^{2} 3 p^{6} 5 f$ & ${ }^{2} \mathrm{~F}_{7 / 2}$ & 927102 & $926734(368)$ \\
\hline 90 & $3 s^{2} 3 p^{6} 6 f$ & ${ }^{2} \mathrm{~F}_{5 / 2}$ & 1016560 & $1015284(1276)$ \\
\hline 91 & $3 s^{2} 3 p^{6} 6 f$ & ${ }^{2} \mathrm{~F}_{7 / 2}$ & 1016570 & $1015322(1248)$ \\
\hline 103 & $3 s^{2} 3 p^{6} 7 f$ & ${ }^{2} \mathrm{~F}_{5 / 2}$ & 1069873 & 1068877 (996) \\
\hline 104 & $3 s^{2} 3 p^{6} 7 f$ & ${ }^{2} \mathrm{~F}_{7 / 2}$ & 1070029 & $1068900(1129)$ \\
\hline
\end{tabular}

Notes. The columns provide: the experimental level energies $E_{\exp }$ $\left(\mathrm{cm}^{-1}\right)$, those for the 23 configuration target (Table 5) $E_{\text {calc }}\left(\mathrm{cm}^{-1}\right)$. Values in parentheses indicate differences with the observed and theoretical energies. The full dataset is available at the CDS. 
Table 7. List of strongest Fe vIII lines in the 50-170 ̊̊ range.

\begin{tabular}{|c|c|c|c|c|c|c|c|}
\hline$i-j$ & Levels & $\begin{array}{l}\text { Int } \\
10^{8} \\
\end{array}$ & $\begin{array}{c}\text { Int } \\
10^{12} \\
\end{array}$ & $g f$ & $A_{j i}\left(\mathrm{~s}^{-1}\right)$ & $\lambda_{\exp }(\AA)$ & $\lambda_{\text {th }}(\AA)$ \\
\hline $2-49$ & $3 p^{6} 3 d^{2} D_{5 / 2}-3 p^{5} 3 d^{2}{ }^{2} D_{5 / 2}$ & 8.6 & 8.5 & 7.06 & $2.8 \times 10^{11}$ & 168.172 & $159.73(-8.4)$ \\
\hline $1-50$ & $3 p^{6} 3 d^{2} D_{3 / 2}-3 p^{5} 3 d^{2}{ }^{2} D_{3 / 2}$ & 6.1 & 5.0 & 4.56 & $2.7 \times 10^{11}$ & 167.486 & $159.20(-8.3)$ \\
\hline $2-48$ & $3 p^{6} 3 d^{2} D_{5 / 2}-3 p^{5} 3 d^{2}{ }^{2} P_{3 / 2}$ & 5.0 & 4.9 & 3.82 & $2.2 \times 10^{11}$ & 168.544 & $162.16(-6.4)$ \\
\hline $1-47$ & $3 p^{6} 3 d^{2} D_{3 / 2}-3 p^{5} 3 d^{2}{ }^{2} P_{1 / 2}$ & 3.1 & 2.5 & 2.10 & $2.4 \times 10^{11}$ & 168.929 & $162.43(-6.5)$ \\
\hline $2-54$ & $3 p^{6} 3 d^{2} D_{5 / 2}-3 p^{6} 4 f^{2} F_{7 / 2}$ & 1.3 & 1.3 & 4.30 & $2.1 \times 10^{11}$ & 131.240 & $129.02(-2.2)$ \\
\hline $1-53$ & $3 p^{6} 3 d^{2} D_{3 / 2}-3 p^{6} 4 f^{2} F_{5 / 2}$ & 1.0 & 0.82 & 2.97 & $1.9 \times 10^{11}$ & 130.941 & $128.71(-2.2)$ \\
\hline $2-50$ & $3 p^{6} 3 d^{2} D_{5 / 2}-3 p^{5} 3 d^{2}{ }^{2} D_{3 / 2}$ & 0.59 & 0.48 & 0.44 & $2.6 \times 10^{10}$ & 168.003 & $159.69(-8.3)$ \\
\hline $1-49$ & $3 p^{6} 3 d^{2} D_{3 / 2}-3 p^{5} 3 d^{2}{ }^{2} D_{5 / 2}$ & 0.52 & 0.52 & 0.43 & $1.7 \times 10^{10}$ & 167.654 & $159.24(-8.4)$ \\
\hline $1-48$ & $3 p^{6} 3 d^{2} D_{3 / 2}-3 p^{5} 3 d^{2}{ }^{2} P_{3 / 2}$ & 0.48 & 0.47 & 0.37 & $2.2 \times 10^{10}$ & 168.024 & $161.65(-6.4)$ \\
\hline $2-84$ & $3 p^{6} 3 d^{2} D_{5 / 2}-3 p^{6} 5 f^{2} F_{7 / 2}$ & 0.20 & 0.20 & 1.40 & $9.9 \times 10^{10}$ & 108.077 & $108.13(0.1)$ \\
\hline $1-83$ & $3 p^{6} 3 d^{2} D_{3 / 2}-3 p^{6} 5 f^{2} F_{5 / 2}$ & 0.16 & 0.13 & 0.98 & $9.3 \times 10^{10}$ & 107.868 & $107.92(0.0)$ \\
\hline $2-53$ & $3 p^{6} 3 d^{2} D_{5 / 2}-3 p^{6} 4 f^{2} F_{5 / 2}$ & $7.3 \times 10^{-2}$ & $5.9 \times 10^{-2}$ & 0.22 & $1.4 \times 10^{10}$ & 131.257 & $129.03(-2.2)$ \\
\hline $2-93$ & $3 p^{6} 3 d^{2} D_{5 / 2}-3 p^{6} 6 f^{2} F_{7 / 2}$ & $6.8 \times 10^{-2}$ & $6.6 \times 10^{-2}$ & 0.73 & $6.3 \times 10^{10}$ & 98.548 & $98.68(0.1)$ \\
\hline $1-92$ & $3 p^{6} 3 d^{2} D_{3 / 2}-3 p^{6} 6 f^{2} F_{5 / 2}$ & $5.1 \times 10^{-2}$ & $4.2 \times 10^{-2}$ & 0.51 & $5.8 \times 10^{10}$ & 98.371 & $98.49(0.1)$ \\
\hline $2-104$ & $3 p^{6} 3 d^{2} D_{5 / 2}-3 p^{6} 7 f^{2} F_{7 / 2}$ & $3.1 \times 10^{-2}$ & $3.0 \times 10^{-2}$ & 0.51 & $4.9 \times 10^{10}$ & 93.616 & $93.72(0.1)$ \\
\hline $1-103$ & $3 p^{6} 3 d^{2} D_{3 / 2}-3 p^{6} 7 f^{2} F_{5 / 2}$ & $2.3 \times 10^{-2}$ & $1.9 \times 10^{-2}$ & 0.35 & $4.5 \times 10^{10}$ & 93.469 & $93.56(0.1)$ \\
\hline
\end{tabular}

Notes. The relative intensities (photons) $\operatorname{Int}=N_{j} A_{j i} / N_{\mathrm{e}}$ are normalised to the strongest transition and were calculated at electron densities of $10^{8}$ and $10^{12} \mathrm{~cm}^{-3}$ and $\log T[\mathrm{~K}]=5$.6. Weighted oscillator strengths $g f$ and $A$-values $\left(\mathrm{s}^{-1}\right)$ are shown. Experimental $\lambda_{\exp }(\AA)$ and theoretical $\lambda_{\text {th }}(\AA)$ wavelengths are included. Values in parentheses indicate differences between the observed and theoretical wavelengths.

Table 8. List of lines in the soft X-rays from quiet Sun observations.

\begin{tabular}{cccccc}
\hline \hline \multicolumn{3}{c}{ Manson (1972) } & \multicolumn{4}{c}{ Behring et al. (1972) } \\
$\begin{array}{c}\text { Wavelength } \\
\AA\end{array}$ & $\begin{array}{c}\text { Flux } \\
10^{6} \mathrm{ph} \mathrm{cm}^{-2} \mathrm{~s}^{-1}\end{array}$ & ID & $\begin{array}{c}\text { Wavelength } \\
\text { ID }\end{array}$ & & \\
\hline 93.02 & 3.8 & & 93.206 & 15 & \\
93.46 & 2.4 & & 93.618 & 30 & \\
93.71 & 3.1 & & 93.838 & 20 & \\
94.04 & 7.3 & Fe x & 93.933 & 20 & \\
94.45 & 1.8 & & 94.016 & 35 & Fe x \\
94.95 & 1.6 & & & & \\
103.58 & 8.5 & Fe IX & & & \\
108.00 & 2.2 & Fe vIII & & & \\
\hline
\end{tabular}

\subsection{Fe Ix}

The strongest line in the soft $\mathrm{X}$-ray spectra is the $3 \mathrm{p}-4 \mathrm{~s}{ }^{1} \mathrm{~S}_{0}-{ }^{1} \mathrm{P}_{1}$ $103.566 \AA$ line, which we use as a reference. First, we note that the use of the R-matrix data in the Fe Ix model ion means that the predicted intensity of this line is about twice that which is obtained when only DW data are used. The relative intensity of this line with e.g. the $3 \mathrm{~d}-4 \mathrm{f}$ transitions suggests that we have about the correct theoretical intensity. The decay from the $4 \mathrm{~s}^{3} \mathrm{P}_{1}$ $105.208 \AA$ is predicted to have half the intensity of the $103.566 \AA$ line at coronal densities, but actually in the solar spectrum the ratio is 0.7 . This could be due to a blend, considering that at high densities the ratio increases to 0.6 , closer to that which is observed (0.75, Lepson et al. 2002). The alternative is that level mixing is not accurate, although all of the different structure runs provide similar values for the radiative data.

Our ion model predicts several $3 \mathrm{~d}-4 \mathrm{f}$ transitions to be strong. Some of them have not been previously identified. We tentatively identify two of them, observed both in solar and laboratory spectra. We assign them the 133.923 and $136.572 \AA$, and notice that identification of these lines with $3 \mathrm{~d}-4 \mathrm{f}$ transitions was already suggested by Lepson et al. (2002) and also by Foster \& Testa (2011). In addition, we identify the $116.803 \AA$ as a self-blend.
The decays from the $4 \mathrm{~d}$ levels were identified by Alexander et al. (1965).

After the $3 p-4 d$ lines, we predict that the strongest transition array is the $3 \mathrm{~d}-5 \mathrm{f}$ one. By comparing EBIT results with calculations using the Hebrew University Lawrence Livermore Atomic Code (HULLAC, Bar-Shalom et al. 2001) Lepson et al. (2002) suggested that there are a number of $5 \mathrm{f}-3 \mathrm{~d}$ transitions around $90 \AA$, in particular three $5 \mathrm{f}-3 \mathrm{~d}$ transitions at $91.98,93.59$ and $94.07 \AA$.

At low coronal densities, we find that only one line should be observable in the solar spectra, the $5-3023 p^{5} 3 d^{3} F_{4}-3 p^{5} 5 f^{3} G_{5}$. At high densities, there are a number of transitions which might become observable. We have searched for a correspondence between our theoretical energy splittings among the $5 \mathrm{f}$ levels and the difference in wavelengths provided by Lepson et al., and found a few possible coincidences. A tentative identification for these lines is provided in Table 9.

The strongest $3 d-5 f$ line would be at $91.98 \AA$ and further blended with two other transitions. The predicted ratio of this line with our reference 3p-4s $103.566 \AA$ line is in excellent agreement with the measurement from Lepson et al. (2002), as Table 9 shows. This is very encouraging. Two other coincidences have been found. Two self-blends contributing to the lines observed at 93.59 and $94.07 \AA$ by Lepson et al. In these cases, the predicted intensities are only half of what was observed. In the solar case, the strongest $91.98 \AA$ line provides only $1 / 3$ of the observed intensity (assuming that our reference line, the $3 p-4 \mathrm{~s}$ $103.566 \AA$ is not in itself blended in the solar spectrum). The other $3 \mathrm{~d}-5 \mathrm{f}$ lines provide very little flux to the observed solar lines. This is not surprising, considering that most lines are noted as blended by Manson. Also, this of course does not mean that in particular conditions, where strong transition-region emission is present, these lines could not be providing a significant contribution to the $94 \AA$ AIA channel.

We searched for a possible identification of the lines from the $3 d-6 f$ transition array, considering that at least one should be observable in the EBIT spectra, but could not find any suitable 
Table 9. Relative intensities for Fe Ix lines.

\begin{tabular}{lccccccc}
\hline \hline Ion & $\lambda_{\text {exp }}(\AA)$ & $\lambda_{\text {th }}(\AA)$ & $R_{\text {obs }}($ Manson 72$)$ & $R_{\text {th }}\left(10^{8}\right)$ & $R_{\text {obs }}($ Lepson 02) & $R_{\text {th }}\left(10^{12}\right)$ & Transition indices \\
\hline Fe IX & 91.98 & $87.61+87.77+87.94$ & 0.6 & 0.21 & 0.25 & 0.27 & $5-302+6-305+7-308$ \\
Fe IX & 93.59 & $89.10+89.30$ & 0.36 & 0.03 & 0.25 & 0.13 & $11-318+12-319$ \\
Fe IX & 94.07 & $89.56+89.68+89.75$ & 0.85 & 0.07 & 0.30 & 0.14 & $8-311+10-316+9-308$ \\
Fe IX & 103.566 & $98.08(-5.5)$ & 1.0 & 1.0 & 1.0 & 1.0 & $1-107$ \\
\hline
\end{tabular}

Notes. Experimental $\lambda_{\exp }(\AA)$ and theoretical $\lambda_{\text {th }}(\AA)$ wavelengths are included. Observed intensities from Manson (1972) and Lepson (2002) expressed relative to the Fe Ix $3 \mathrm{p}^{6}{ }^{1} \mathrm{~S}_{0}-3 \mathrm{p}^{5} 4 \mathrm{~s}{ }^{1} \mathrm{P}_{1}$ intensity are shown. Theoretical intensities at $10^{8} \mathrm{~cm}^{-3}$ and at $10^{12} \mathrm{~cm}^{-3}$ expressed relative to the Fe Ix $3 \mathrm{p}^{6}{ }^{1} \mathrm{~S}_{0}-3 \mathrm{p}^{5} 4 \mathrm{~s}^{1} \mathrm{P}_{1}$ intensity are also included.

candidate. Further work is needed to identify these lines, as well as other ones such as the $3 \mathrm{~d}-5 \mathrm{p}$.

It is interesting to note that many of the strongest lines, even at relatively low densities (quiet Sun corona), are decays to metastable levels. Indeed, many of the transitions, including the strongest of the $3 \mathrm{~d}-5 \mathrm{f}$, are from levels which become mainly populated through excitation from a metastable one, and not from the ground state. For example, the intensity of the $3 p^{5}$ $3 d^{3} F_{4}-3 p^{5} 5 f^{3} G_{5}$ line becomes four times weaker if only the excitation from the ground state is included in the model.

We have compared our results for Fe IX with those presented by Foster \& Testa (2011) at the same density and temperature values and have found that their results are in reasonable agreement with ours.

We compare the intensities of the $3 d-4 f$ lines using the intensities listed in Datla et al. (1975, first case), which included corrections due to optical depth effects. The plasma source was measured to have $N_{\mathrm{e}}=10^{16.07} \mathrm{~cm}^{-3}$ and $\log T_{\mathrm{e}}[\mathrm{K}]=6.04$. We make use of the "emissivity ratio" technique (see Del Zanna et al. 2004, for details), whereby the observed intensity of a line is divided by its emissivity:

$F_{j i}=\frac{I_{\mathrm{ob}} N_{\mathrm{e}} C}{h v N_{j}\left(N_{\mathrm{e}}, T_{\mathrm{e}}\right) A_{j i}}$

calculated at a fixed electron density $N_{\mathrm{e}}$ (in this case $10^{16.07} \mathrm{~cm}^{-3}$ ) and plotted as a function of the temperature $T_{\mathrm{e}}$. $I_{\mathrm{ob}}$ is the observed intensity in the line, $h$ is Planck's constant, $v$ is the frequency of the line and $N_{j}$ is the number density of the upper level $\mathrm{j}$ of the emitting ion. The scaling constant $C$ is chosen so the curves are close to unity. The emissivity ratios (see Fig. 4) show good agreement to within $\pm 30 \%$, with one exception, between theory and experiment for the $3 s^{2} 3 p^{6}-3 d, 3 s^{2} 3 p^{6}-4 s$, and $3 \mathrm{~d}-4 \mathrm{f}$ transitions, thus giving us confidence in the reliability of the present calculations for the $4 \mathrm{f}$ levels.

\subsection{Fe vIII}

We compare the intensities of the $3 d-4 f, 3 d-5 f$ lines using the intensities listed in Datla et al. (1975, second case). The level populations are in the high density limit and therefore have no relative sensitivity to changes in electron density. As the relative variation in temperature is greater than that in density the emissivity ratio has been plotted as function of the temperature and not of the density. The emissivity ratios show very good agreement between theory and experiment to within $\pm 20 \%$ for the $3 d-3 d, 3 d-4 f$, and $3 d-5 f$ transitions, thus giving us confidence in the reliability of the present calculations for the $5 \mathrm{f}$ levels (see Fig. 5). The contribution of missing resonances in the collision strengths for levels $n \geq 5$ is in fact not expected to be large.

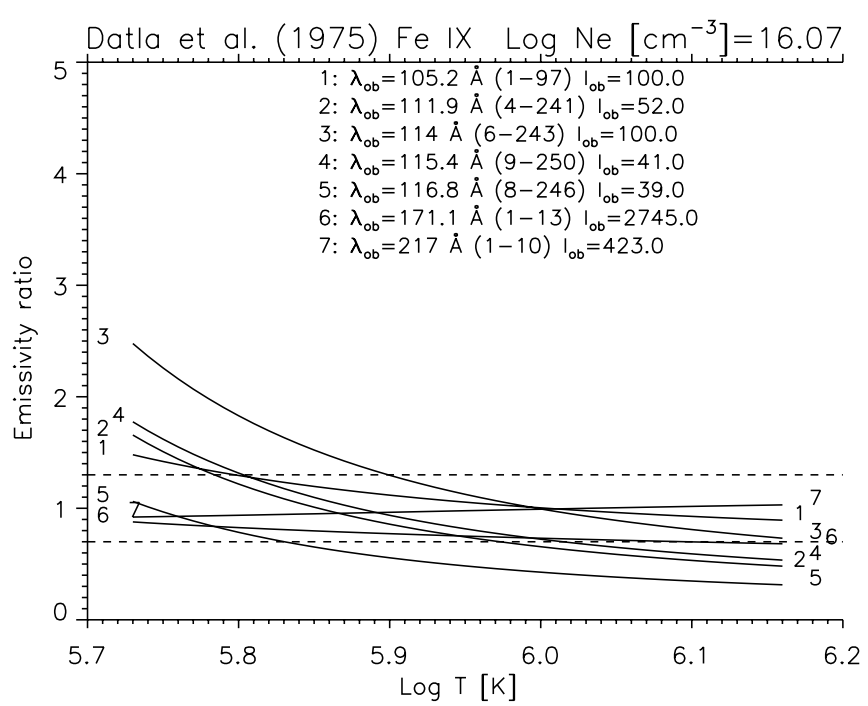

Fig. 4. The emissivity ratio curves from the calibrated theta-pinch laboratory spectra of Datla et al. (1975). $I_{\mathrm{ob}}$ indicates the measured line intensity. Dashed lines at $\pm 30 \%$ have been added to give an indication of the agreement.

We then consider the $3 \mathrm{~d}-5 \mathrm{f}, 3 \mathrm{~d}-6 \mathrm{f}, 3 \mathrm{~d}-7 \mathrm{f}$ transitions. Table 10 shows the relative intensities, predicted and observed. We find an excellent agreement between the relative ratios as observed in the laboratory by Ramonas \& Ryabtsev (1980), which is very encouraging. We find some differences with the corresponding intensities predicted using the excitation data from Czyzak \& Krueger (1966).

The two Fe vIII $3 \mathrm{p}^{6} 3 \mathrm{~d}^{2} \mathrm{D}_{3 / 2}-3 \mathrm{p}^{6} 7 \mathrm{f}^{2} \mathrm{~F}_{5 / 2}$ (93.56 $\AA$ ) and $3 p^{6} 3 d^{2} D_{5 / 2}-3 p^{6} 7 f^{2} F_{7 / 2}(93.72 \AA)$ transitions are within the SDO AIA $94 \AA$ filter, together with the above Fe Ix lines, and both the Fe XVIII (93.923 $\AA$ ) transition from $2 \mathrm{~s} 2 \mathrm{p}^{6}{ }^{1} \mathrm{~S}_{1 / 2}-2 \mathrm{~s}^{2} 2 \mathrm{p}^{5}$ ${ }^{3} \mathrm{P}_{3 / 2}$ and the Fex (94.012 ̊) $3 \mathrm{~s}^{2} 3 \mathrm{p}^{4} 4 \mathrm{~s}^{2} \mathrm{D}_{5 / 2}-3 \mathrm{~s}^{2} 3 \mathrm{p}^{5}{ }^{2} \mathrm{P}_{3 / 2}$. Del Zanna et al. (2011) used the approximate excitation data from Czyzak \& Krueger (1966), included in CHIANTI v6.0, to show that in particular conditions (at the footpoints of $1 \mathrm{MK}$ loops) these $7 \mathrm{f}-3 \mathrm{~d}$ lines are expected to provide a considerable contribution to the photons recorded in this band, so we can confirm here that to be the case. However, as Table 10 shows, there is plenty of unidentified flux in quiet Sun conditions, i.e. the $7 \mathrm{f}-$ $3 \mathrm{~d}$ transitions only provide a small fraction of the observed lines contributing to the $94 \AA$ AIA channel.

\section{Conclusions}

The new atomic calculations presented here provide a significant advance over previous work. We have extended existing 
Table 10. Relative intensities for Fe vIII lines.

\begin{tabular}{lccccc}
\hline \hline Ion & $\lambda_{\text {exp }}(\AA)$ & $R_{\text {obs }}($ Manson 72$)$ & $R_{\text {obs }}($ Ramonas \& Ryabtsev 80$)$ & $R_{\text {th }}\left(10^{8}-10^{12}\right)$ & Transition indices \\
\hline Fe VIII & 93.469 & 1.1 & 0.07 & 0.06 & $1-103$ \\
Fe VIII & 93.616 & 1.4 & 0.09 & 0.09 & $2-104$ \\
Fe VIII & $98.371+98.548$ & 2.8 & 0.33 & 0.33 & $1-92+2-93$ \\
Fe VIII & $107.868+108.077$ & 1.0 & 1.0 & 1.0 & $1-83+2-84$ \\
\hline
\end{tabular}

Notes. Experimental wavelengths $\lambda_{\exp }(\AA)$ are included. Observed intensities from Manson (1972) and Ramonas \& Ryabtsev (1980) expressed relative to the combined Fe vIII $3 \mathrm{~d}^{2} \mathrm{D}_{3 / 2}-5 \mathrm{f}^{2} \mathrm{~F}_{5 / 2} \& 3 \mathrm{~d}^{2} \mathrm{D}_{5 / 2}-5 \mathrm{f}^{2} \mathrm{~F}_{7 / 2}$ intensities are shown. Theoretical intensities at $10^{8} \mathrm{~cm}^{-3}$ and $10^{12} \mathrm{~cm}^{-3}$ expressed relative to the combined Fe vIII $3 \mathrm{~d}^{2} \mathrm{D}_{3 / 2}-5 \mathrm{f}^{2} \mathrm{~F}_{5 / 2} \& 3 \mathrm{~d}^{2} \mathrm{D}_{5 / 2}-5 \mathrm{f}^{2} \mathrm{~F}_{7 / 2}$ intensities are also included.

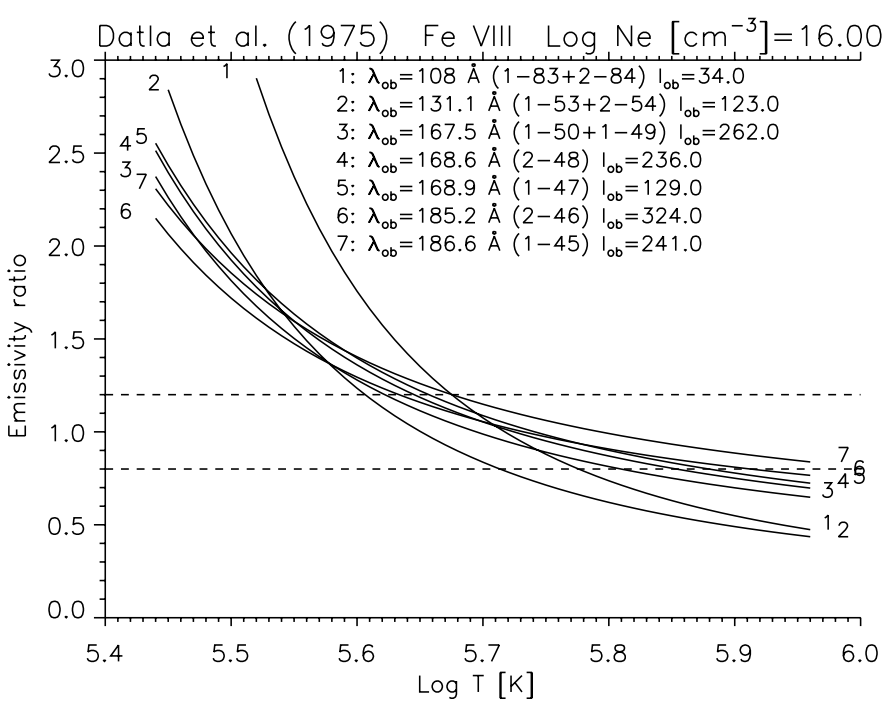

Fig. 5. The emissivity ratio curves from the calibrated theta-pinch laboratory spectra of Datla et al. (1975). $I_{\mathrm{ob}}$ indicates the measured line intensity. Dashed lines at $\pm 20 \%$ have been added to give an indication of the agreement.

calculations of energy levels, radiative transition rates and collisional excitation rates up to $n=6$ for Fe IX and $n=7$ for Fe vIII. The new data allow the calculation of line intensities. We have provided several new identifications, but many other lines are still unknown.

This paper is just a first step into the complex area of identifying and providing atomic data for lines in the crowded 50$170 \AA$ A spectral region. Jordan (1968) used collisional excitation rates estimated by one of us (PJS) to suggest that e.g. for Fe IX transitions from $3 \mathrm{p}^{5} 4 l(l=\mathrm{s}, \mathrm{p}, \mathrm{d}, \mathrm{f})$ to either the ground state or the $3 p^{5} 3 d$ would contribute significantly to the observed lines. A large number of lines present in this region have yet to be identified, however we do find for some transitions a relatively good agreement with observations.

Based on our calculations the predicted contribution of Fe IX $5 f-3 d$ and Fe vIII $7 f-3 d$ transitions is only a small fraction of the observed lines in the 93-95 A region for quiet Sun conditions. As a result the contribution of these lines to the SDO/AIA $94 \AA$ filter is predicted to be small.

Acknowledgements. The authors thank Dr. Adam Foster \& Dr. Paola Testa for providing their Fe IX atomic data for comparison with our own. The authors acknowledge the support of STFC. B.O.D. was supported by funding from the Gates Cambridge Trust. CHIANTI is a collaborative project involving researchers, at the Universities of: Cambridge (UK), George Mason (USA), and Michigan (USA). This work was carried out as part of the UK APAP project.

\section{References}

Aggarwal, K. M., Keenan, F. P., Kato, T., \& Murakami, I. 2006, A\&A, 460, 331 Alexander, E., Feldman, U., \& Fraenkel, B. S. 1965, J. Opt. Soc. Am., 55, 650 Aschwanden, M. J., \& Boerner, P. 2011, ApJ, in press

Badnell, N. R. 1997, J. Phys. B: At. Mol. Opt. Phys., 30, 1

Badnell, N. R. 2011, Comp. Phys. Commun., 182, 1528

Bar-Shalom, A., Klapisch, M., \& Oreg, J. 2001, J. Quant. Spec. Radiat. Transf., 71, 169

Behring, W. E., Cohen, L., \& Feldman, U. 1972, ApJ, 175, 493

Beiersdorfer, P., Lepson, J. K., Brown, G. V., et al. 1999, ApJ, 519, L185

Brinkman, A. C., Gunsing, C. J. T., Kaastra, J. S., et al. 2000, ApJ, 530, L111

Culhane, J. L., Harra, L. K., James, A. M., et al. 2007, Sol. Phys., 60

Czyzak, S. J., \& Krueger, T. K. 1966, ApJ, 144, 381

Datla, R. U., Blaha, M., \& Kunze, H.-J. 1975, Phys. Rev. A, 12, 1076

Del Zanna, G. 2009, A\&A, 508, 513

Del Zanna, G., Berrington, K. A., \& Mason, H. E. 2004, A\&A, 422, 731

Del Zanna, G., O’Dwyer, B., \& Mason, H. E. 2011, A\&A, accepted

Dere, K. P., Landi, E., Young, P. R., et al. 2009, A\&A, 498, 915

Eissner, W. 1998, Comp. Phys. Commun., 114, 295

Eissner, W., Jones, M., \& Nussbaumer, H. 1974, Comp. Phys. Commun., 8, 270

Fawcett, B. C., Peacock, N. J., \& Cowan, R. D. 1968, J. Phys. B: At. Mol. Phys., 1,295

Fawcett, B. C., Kononov, E. Y., Hayes, R. W., \& Cowan, R. D. 1972, J. Phys. B: At. Mol. Phys., 5, 1255

Foster, A. R., \& Testa, P. 2011, ApJ, 740, L52

Gabriel, A. H., \& Fawcett, B. C. 1965, Nature, 206, 390

Griffin, D. C., Pindzola, M. S., \& Badnell, N. R. 2000, A\&AS, 142, 317

Gu, M. F. 2003, ApJ, 582, 1241

Jordan, C. 1968, J. Phys. B: At. Mol. Phys., 1, 1004

Kruger, P. G., \& Weissberg, S. G. 1937, Phys. Rev., 52, 314

Kruger, P. G., Weissberg, S. G., \& Phillips, L. W. 1937, Phys. Rev., 51, 1090

Landi, E., \& Young, P. R. 2009, ApJ, 707, 1191

Lemen, J., Title, A., Akin, D., et al. 2011, Sol. Phys., submitted

Lepson, J. K., Beiersdorfer, P., Brown, G. V., et al. 2002, ApJ, 578, 648

Liang, G. Y., \& Badnell, N. R. 2011, A\&A, 528, A69

Liang, G. Y., Badnell, N. R., Zhao, G., Zhong, J. Y., \& Wang, F. L. 2011, A\&A, 533, A87

Manson, J. E. 1972, Sol. Phys., 27, 107

O’Dwyer, B., Del Zanna, G., Mason, H. E., Weber, M. A., \& Tripathi, D. 2010, A\&A, 521, A21

Ramonas, A. A., \& Ryabtsev, A. N. 1980, Optics and Spectroscopy, 48, 348

Storey, P. J., Zeippen, C. J., \& Le Dourneuf, M. 2002, A\&A, 394, 753

Summers, H. P. 2006, The ADAS User Manual v2.6,

http://www .adas.ac.uk/manual.php

Verma, N., Jha, A. K. S., \& Mohan, M. 2006, ApJS, 164, 297

Wagner, W. J., \& House, L. L. 1971, ApJ, 166, 683

Woods, T. N., Eparvier, F. G., Hock, R., et al. 2010, Sol. Phys., DOI: 10.1007/S11207-009-9487-6

Young, P. R. 2009, ApJ, 691, L77

Young, P. R., \& Landi, E. 2009, ApJ, 707, 173

Zeng, J., Jin, F., Zhao, G., \& Yuan, J. 2003, J. Phys. B: At. Mol. Opt. Phys., 36, 3457 